\title{
Comprehensive Analysis of NAC Domain Transcription Factor Gene Family in Populus trichocarpa
}

\author{
Ruibo $\mathrm{Hu}^{1 \dagger}$, Guang $\mathrm{Qi}^{1 \dagger}$, Yingzhen Kong ${ }^{1,2 \dagger}$, Dejing Kong ${ }^{1}$, Qian Gao ${ }^{1}$, Gongke Zhou ${ }^{{ }^{*}}$
}

\begin{abstract}
Background: NAC (NAM, ATAF1/2 and CUC2) domain proteins are plant-specific transcriptional factors known to play diverse roles in various plant developmental processes. NAC transcription factors comprise of a large gene family represented by more than 100 members in Arabidopsis, rice and soybean etc. Recently, a preliminary phylogenetic analysis was reported for NAC gene family from 11 plant species. However, no comprehensive study incorporating phylogeny, chromosomal location, gene structure, conserved motifs, and expression profiling analysis has been presented thus far for the model tree species Populus.

Results: In the present study, a comprehensive analysis of NAC gene family in Populus was performed. A total of 163 full-length NAC genes were identified in Populus, and they were phylogeneticly clustered into 18 distinct subfamilies. The gene structure and motif compositions were considerably conserved among the subfamilies. The distributions of 120 Populus NAC genes were non-random across the 19 linkage groups (LGs), and 87 genes (73\%) were preferentially retained duplicates that located in both duplicated regions. The majority of NACs showed specific temporal and spatial expression patterns based on EST frequency and microarray data analyses. However, the expression patterns of a majority of duplicate genes were partially redundant, suggesting the occurrence of subfunctionalization during subsequent evolutionary process. Furthermore, quantitative real-time RT-PCR (RT-qPCR) was performed to confirm the tissue-specific expression patterns of 25 NAC genes.

Conclusion: Based on the genomic organizations, we can conclude that segmental duplications contribute significantly to the expansion of Populus NAC gene family. The comprehensive expression profiles analysis provides first insights into the functional divergence among members in NAC gene family. In addition, the high divergence rate of expression patterns after segmental duplications indicates that NAC genes in Populus are likewise to have been retained by substantial subfunctionalization. Taken together, our results presented here would be helpful in laying the foundation for functional characterization of NAC gene family and further gaining an understanding of the structure-function relationship between these family members.
\end{abstract}

\section{Background}

Transcriptional regulation of gene expression controls many important cellular processes in plants, such as cellular morphogenesis, signaling transduction and environmental stress responses [1]. Transcription factors (TFs) are types of proteins that regulate gene expression by binding to specific cis-acting promoter elements, thereby activating or repressing the transcriptional rates

\footnotetext{
* Correspondence: zhougk@qibebt.ac.cn

† Contributed equally

${ }^{1}$ Qingdao Institute of BioEnergy and Bioprocess Technology, Chinese Academy of Sciences, Qingdao 266101, PR China
}

of their target genes [1,2]. Thus, the identification and functional characterization of TFs is essential for the reconstruction of transcriptional regulatory networks [3]. In plants, totally more than 50 different families of TFs have been identified based on bioinformatics analysis so far [1,3]. The Arabidopsis genome encodes at least 1550 TFs, accounting for about $6.2 \%$ of its estimated total number of gene [4]. As for Populus, about $6.4 \%$ of its genome was found to encode more than $2900 \mathrm{TFs}$ $[3,5]$.

NAC (NAM, ATAF1/2 and CUC2) domain proteins comprise of one of the largest plant-specific TFs

\section{Biomed Central}


represented by $\sim 105$ genes in Arabidopsis [6], $\sim 140$ genes in rice [7] and $\sim 101$ genes in soybean genome [8]. NAC proteins commonly possess a conserved NAC domain at the $\mathrm{N}$-terminus, which comprises nearly 160 amino acid residues that are divided into five subdomains (A-E) $[6,9,10]$. A sequence region of 60 residues within the NAC domain contains a unique TF fold consisting of a twisted-sheet bounded by a few helical elements [11]. NAC domain has been implicated in nuclear localization, DNA binding, and the formation of homodimers or heterodimers with other NAC domain proteins [11-16]. In contrast, the $C$-terminal regions of NAC proteins are highly divergent [6-9,14], and confer the regulation diversities of transcriptional activation activity $[11,13,15-22]$.

NAC proteins have been implicated to participate in a wide range of plant developmental processes, including shoot apical meristem development [10,23-26], floral morphogenesis [27], lateral root development [15,28], leaf senescence $[29,30]$, stress inducible flowering induction [31,32], embryo development [11], cell cycle control [33-35], hormone signaling $[15,18,33,36]$, grain nutrient remobilization $[30,37]$ and shoot branching determination [38]. Particularly, numerous NAC domain proteins have also been implicated in plant abiotic stresses and defense responses such as drought, salinity, cold shock, mechanical wounding and viral infection $[18,20,28$, 36,39-47]. Recently, accumulating evidences indicated that a considerable portion of NAC domain proteins also play crucial roles in the processes of xylogenesis, fiber development, and wood formation in vascular plants [17,19,21,48-55].

In Arabidopsis, evidences indicated that a subset of closely related NAC domain proteins including NST1/ ANAC043 (NAC Secondary Wall Thickening Promoting Factor 1), NST2/ANAC066, and NST3/SND1(Secondary Wall-associated NAC Domain Protein)/ANAC012 act as master transcriptional switches governing secondary cell wall biosynthesis in a partially redundant manner [52-55]. NST1/ANAC043 and NST2/ANAC066 were shown to function redundantly in secondary cell wall thickening in anther endothecium and induced ectopic secondary wall thickenings in various tissues when expressed ectopically $[52,55]$, whereas NST1 and NST3/SND1/ANAC012 redundantly regulate the secondary wall thickening in inter-fascicular fiber of inflorescence stems and secondary xylem of hypocotyls in Arabidopsis [53,54]. SND2/ ANAC073 and SND3/ANAC010 have also been shown to function in the formation of secondary cell wall in fibers, and their dominant repression resulted in remarkable reduction in the secondary wall thickening [51]. Interestingly, both of SND2 and SND3 seem to function at downstream of NST1 and NST3/SND1, whereas SND2 was identified as a direct target of NST3/SND1 [51]. VND6 and VND7 (Vascular-related NAC-Domain) have been proposed to be regulators of the formation of vascular vessels in Arabidopsis $[21,56]$. They act as key transcriptional switches regulating the differentiation of metaxylem and protoxylem, respectively, in primary roots $[21,56]$. Consequently, NSTs may activate the secondary wall biosynthetic program in fibers, whereas VNDs are proposed to specifically regulate secondary wall biosynthesis in vascular vessels [51]. Another XND1/ANAC104 (Xylem NAC Domain 1) gene has been revealed to influence the differentiation of tracheary elements and xylem development in Arabidopsis by negatively regulating terminal secondary wall biosynthesis and programmed cell death in xylem vessel cells.

Although quite a few NAC TFs have been functionally characterized in model plants Arabidopsis and rice, the functions of majority of NAC members remain unknown. Especially in Populus, the typical model tree species, there are only very limited reports on the functional characterization of NAC TFs. Recently, Zhong et al (2009) reported the molecular cloning and functional characterization of six NAC genes in Populus [19]. Among the six NAC genes, WND2B (Wood-Associated NAC Domain Transcription Factors) and WND6B effectively complemented the secondary wall defects in snd1/ nst 1 double mutant, and when ectopically overexpressed in Arabidopsis, they induced ectopic deposition of secondary walls. These findings demonstrated that WND2B and WND6B genes are functional orthologs of Arabidopsis SND1 and master switches activating secondary wall biosynthesis during wood formation in Populus [19]. Shen et al (2009) carried out a genomewide bioinformatics survey on plant NAC domain TFs and identified a total of 1,232 NAC proteins from 11 different plant species including 148 NAC TFs from Populus [57]. However, only sequence phylogeny analysis of Populus NAC TFs was performed in their report and no detailed analysis including genome organization, gene structure and expression compendium have been conducted [57]. In the present study, we further performed a genome-wide identification of NAC domain TFs in Populus and revealed an expanded NAC family with totally 163 members. Detailed analysis including sequence phylogeny, genome organization, gene structure, conserved motifs and expression profiling was performed. It is noteworthy that a subset of more than thirty NAC genes showed the highest level of transcript abundance in differentiating xylem and cambium tissues. Among them, twenty-five genes were further investigated for their tissue-specific expressions by quantitative real-time RT-PCR (RT-qPCR) analysis. Our results may provide a subset of potential candidate NAC genes for future engineering modification of lignocellulosic biomass characteristics in Populus. 


\section{Results and Discussion}

\section{Identification of NAC gene family in Populus}

The NAC domain genes are plant-specific TFs presented as one of the largest multigene families in Arabidopsis, rice, soybean, maize, grape and sorghum etc [3,6,8,57]. Hidden Markov Model (HMM) profile of the Pfam NAC domain (PF02365) was exploited as query to identify the NAC genes in Populus genome (release 2.0, http://www.phytozome.net/poplar). Initially, a total of 170 nonredundant putative NAC genes were identified. In an attempt to demonstrate the reliability of the identified genes, keyword search with NAM against NCBI nucleotide database was performed, resulting in 153 members which were all included in the NACs identified above. In comparison to the NAC gene family in PlnTFDB http://plntfdb.bio.uni-potsdam.de/v3.0/[3] and DPTF http://dptf.cbi.pku.edu.cn/[58], in which 167 and 172 members were proposed respectively, the 170 gene loci revealed in the present study were roughly in agreement with the former. Then, the discrepancy loci identified in our studies with the two databases mentioned above were further scrutinized to see if any misannotations were inferred. Among them, sixteen genes may represent putative pseudogenes or incorrect annotations, and manual reannotation was performed to correct and reannotate the putative NACs using online web server FGENESH http://linux1.softberry.com/berry.phtml[59]. In this endeavor, seven sequences encoding only truncated proteins were excluded from further analysis. In addition, we combined the newly released genome annotations with the results inferred by FGENESH to make the annotations more convincing. Finally, all the putative 163 NAC genes were further manually analyzed using InterProScan program http://www.ebi.ac.uk/Tools/ InterProScan/ to confirm the presence of NAM domain [60]. In a recently published report, a total of 148 NAC genes were identified in Populus by a genome-wide bioinformatics survey [57]. In this study, we further revealed an expanded NAC family in Populus with totally 163 members. We designated Populus NAC genes as PNAC following the nomenclature proposed in the previous study [6]. The identified NAC genes in Populus encode proteins ranging from 117 to 718 amino acids (aa) in length with an average of 342 aa. Remarkably, in most cases, two or more Populus NAC genes were found for every ortholog in Arabidopsis. The detailed information of NAC family genes in Populus, including accession numbers and similarities to their Arabidopsis orthologs as well as nucleotide and protein sequences was listed in Table 1 and Additional file 1.

The NAC gene family in Populus is by far the largest one compared to the estimates for other plant species, which range from $\sim 105$ in Arabidopsis [6], 140 in rice
[7] and $\sim 101$ in soybean [8]. The member of NAC genes in Populus is roughly 1.58 fold than that of Arabidopsis, which is in consistency with the ratio of $1.4 \sim 1.6$ putative Populus homologs for each Arabidopsis gene based on comparative genomics studies [5]. In comparison to its closest woody perennial grape (Vitis vinifera), which possesses only about 79 NAC genes [3], the NAC genes in Populus seems to be highly expanded [5]. It can be speculated that the presence of more NAC genes in Populus genome may reflect the great needs for these genes to involve in the complicated transcriptional regulations in this woody perennial species. This expansion appears to be arisen from multiple gene duplication events, including a whole-genome duplication event in the Populus lineage followed by multiple segmental and tandem duplication events [5].

\section{Phylogenetic analysis of NAC gene family in Populus, Arabidopsis and rice}

To examine the phylogenetic relationship among the NAC domain proteins in Populus, Arabidopsis and rice, an unrooted tree was constructed from alignments of the full-length NAC protein sequences (Figure 1). The phylogenetic tree was constructed using MEGA 4.0 by employing the Neighbor-Joining (NJ), Minimal Evolution (ME) and Maximum Parsimony (MP) methods, respectively. The tree topologies produced by the three algorithms were largely comparable with only minor modifications at interior branches (data not shown). Therefore, only the NJ phylogenetic tree was subject to further analysis in our study. Moreover, we constructed the phylogenetic tree with the conserved N-terminal NAC domains A to $\mathrm{E}$ using the same algorithm, which was largely consistent to the phylogenetic analyses performed by the previous studies [57] (see details in Additional file 2). As indicated in Figure 1, the phylogenetic tree divided the NAC family proteins into 18 distinct subgroups. PNAC109 was distinguished from other NAC members and formed an individual clade with a robust bootstrap value support (99\%) (Figure 1 and Additional file 3). For simplicity, the subgroups were designated as alphabetical families (NAC-a to NAC-r) based on tree topologies. Although the bootstrap values were somewhat low due to the large number of sequences, which was also presented in previous studies [61-65], more significant bootstrap values in the distal branches allowed us to group the Populus, Arabidopsis and rice NAC proteins into distinct families (Additional file 3). Moreover, we sought other evidences such as gene structure, motif compositions and expression patterns as described below to support the reliability of the subgroup classification.

In general, the NAC members demonstrated an interspersed distribution in majority of subfamilies, indicating 
Table 1 NAC gene family in Populus

\begin{tabular}{|c|c|c|c|c|c|}
\hline Gene symbol & Gene locus & Arabidopsis ortholog locus & Arabidopsis locus description & Score & E-value \\
\hline PNAC001 & POPTR_0009s05700.1 & AT1G01720.1 & ANAC002, ATAF1 & 179 & $3 e-045$ \\
\hline PNAC002 & POPTR_0018s10310.1 & AT1G01720.1 & ANAC002, ATAF1 & 187 & $8 e-048$ \\
\hline PNAC003 & POPTR_0017s09630.1 & AT3G04070.2 & ANAC002, ATAF1 & 146 & $2 \mathrm{e}-048$ \\
\hline PNAC004 & POPTR_0005s20240.1 & AT1G01720.1 & ANAC002, ATAF1 & 392 & $1 e-109$ \\
\hline PNAC005 & POPTR_0005s07060.1 & AT1G01720.1 & ANAC002, ATAF1 & 357 & $5 e-099$ \\
\hline PNAC006 & POPTR_0002s08150.1 & AT1G01720.1 & ANAC002, ATAF1 & 416 & $1 e-117$ \\
\hline PNAC007 & POPTR_0007s04780.1 & AT1G01720.1 & ANAC002, ATAF1 & 366 & $1 e-101$ \\
\hline PNAC008 & POPTR_0015s14770.1 & AT1G12260.1 & ANAC007, VND4 & 374 & $1 e-104$ \\
\hline PNAC009 & POPTR_0003s11250.1 & AT1G12260.1 & ANAC007, VND4 & 436 & $1 e-122$ \\
\hline PNAC010 & POPTR_0012s14660.1 & AT1G12260.1 & ANAC007, VND4 & 340 & 7e-094 \\
\hline PNAC011 & POPTR_0001s00220.1 & AT1G12260.1 & ANAC007, VND4 & 449 & $1 e-127$ \\
\hline PNAC012 & POPTR_0010s13980.1 & AT1G25580.1 & ANAC008 & 476 & $1 e-134$ \\
\hline PNAC013 & POPTR_0008s11550.1 & AT1G25580.1 & ANAC008 & 509 & $1 e-144$ \\
\hline PNAC014 & POPTR_0017s11830.1 & AT1G26870.1 & ANAC009 & 299 & $2 \mathrm{e}-081$ \\
\hline PNAC015 & POPTR_0004s12850.1 & AT1G26870.1 & ANAC009 & 310 & $2 e-084$ \\
\hline PNAC016 & POPTR_0004s12850.1 & AT1G26870.1 & ANAC009 & 100 & $9 e-022$ \\
\hline PNAC017 & POPTR_0001s45250.1 & AT1G32770.1 & ANAC012, SND1, NST3 & 294 & $6 e-080$ \\
\hline PNAC018 & POPTR_0011s15230.1 & AT1G33060.1 & ANAC014 & 420 & $1 e-117$ \\
\hline PNAC019 & POPTR_0011s15230.1 & AT1G33060.2 & ANAC014 & 431 & $1 e-121$ \\
\hline PNAC020 & POPTR_0014s07220.1 & AT1G33060.2 & ANAC014 & 103 & $3 e-022$ \\
\hline PNAC021 & POPTR_0002s15550.1 & AT1G33060.1 & ANAC014 & 135 & $9 e-032$ \\
\hline PNAC022 & POPTR_0014s07220.1 & AT1G33060.1 & ANAC014 & 109 & $4 \mathrm{e}-024$ \\
\hline PNAC023 & POPTR_0002s15530.1 & AT1G33060.2 & ANAC014 & 154 & $1 e-037$ \\
\hline PNAC024 & POPTR_0012s06340.1 & AT1G34190.1 & ANAC017 & 117 & $2 \mathrm{e}-027$ \\
\hline PNAC025 & POPTR_0043s00220.1 & AT1G34190.1 & ANAC017 & 140 & $2 e-034$ \\
\hline PNAC026 & POPTR_0013s10770.1 & AT1G34190.1 & ANAC017 & 140 & $2 e-034$ \\
\hline PNAC027 & POPTR_0043s00220.1 & AT1G34190.1 & ANAC017 & 180 & $4 e-046$ \\
\hline PNAC028 & POPTR_0005s22180.1 & AT1G34190.1 & ANAC017 & 441 & $1 e-124$ \\
\hline PNAC029 & POPTR_0002s06210.1 & AT1G34190.1 & ANAC017 & 432 & $1 e-121$ \\
\hline PNAC030 & POPTR_0014s07210.1 & AT4G35580.2 & ANAC018, NAM & 157 & $4 e-039$ \\
\hline PNAC031 & POPTR_0014s10350.1 & AT4G35580.2 & ANAC018, NAM & 180 & $9 e-046$ \\
\hline PNAC032 & POPTR_0014s10350.1 & AT4G35580.2 & ANAC018, NAM & 181 & $5 e-046$ \\
\hline PNAC033 & POPTR_0002s18310.1 & AT4G35580.2 & ANAC018, NAM & 184 & $3 e-047$ \\
\hline PNAC034 & POPTR_0002s18320.1 & AT4G35580.2 & ANAC018, NAM & 144 & $2 \mathrm{e}-034$ \\
\hline PNAC035 & POPTR_0002s17700.1 & AT4G35580.2 & ANAC018, NAM & 130 & $2 \mathrm{e}-030$ \\
\hline PNAC036 & POPTR_0002s15560.1 & AT4G35580.2 & ANAC018, NAM & 171 & 4e-093 \\
\hline PNAC037 & POPTR_0014s10320.1 & AT4G35580.2 & ANAC018, NAM & 138 & $8 e-033$ \\
\hline PNAC038 & POPTR_0014s10320.1 & AT4G35580.2 & ANAC018, NAM & 129 & $9 e-031$ \\
\hline PNAC039 & POPTR_0010s18180.1 & AT1G54330.1 & ANAC020 & 290 & $9 e-079$ \\
\hline PNAC040 & POPTR_0008s08100.1 & AT1G54330.1 & ANAC020 & 316 & $1 e-086$ \\
\hline PNAC041 & POPTR_0005s10100.1 & AT1G56010.2 & ANAC021/22, AtNAC1 & 323 & $7 e-089$ \\
\hline PNAC042 & POPTR_0007s08420.1 POPTR_0007s08430.1 & AT1G56010.2 & ANAC021/22, AtNAC1 & 337 & $5 e-093$ \\
\hline PNAC043 & POPTR_0004s04920.1 & AT1G61110.1 & ANAC025 & 232 & $3 e-061$ \\
\hline PNAC044 & POPTR_0016s05580.1 & AT1G61110.1 & ANAC025 & 137 & $2 \mathrm{e}-032$ \\
\hline PNAC045 & POPTR_0016s08970.1 & AT1G61110.1 & ANAC025 & 205 & $2 \mathrm{e}-053$ \\
\hline PNAC046 & POPTR_0006s13140.1 & AT1G61110.1 & ANAC025 & 247 & $7 e-066$ \\
\hline PNAC047 & POPTR_0011s04650.1 & AT1G61110.1 & ANAC025 & 339 & $1 e-093$ \\
\hline PNAC048 & POPTR_0004s03820.1 & AT1G61110.1 & ANAC025 & 332 & 3e-091 \\
\hline PNAC049 & POPTR_0017s02020.1 & AT1G65910.1 & ANAC028 & 464 & $1 e-130$ \\
\hline PNAC050 & POPTR_0004s07910.1 & AT1G65910.1 & ANAC028 & 465 & $1 e-131$ \\
\hline PNAC051 & POPTR_0011s12190.1 & AT1G65910.1 & ANAC028 & 68 & $1 e-011$ \\
\hline
\end{tabular}


Table 1: NAC gene family in Populus (Continued)

\begin{tabular}{|c|c|c|c|c|c|}
\hline PNAC052 & POPTR_0014s04080.1 & AT1G65910.1 & ANAC028 & 73 & $1 \mathrm{e}-013$ \\
\hline PNAC053 & POPTR_0010s17350.1 & AT1G69490.1 & ANAC029, ATNAP, NAP & 305 & 2e-083 \\
\hline PNAC054 & POPTR_0003s10250.1 & AT1G69490.1 & ANAC029, ATNAP, NAP & 119 & $3 e-027$ \\
\hline PNAC055 & POPTR_0014s02580.1 & AT1G69490.1 & ANAC029, ATNAP, NAP & 89 & $4 e-018$ \\
\hline PNAC056 & POPTR_0019s11330.1 & AT1G71930.1 & ANAC030, VND7 & 249 & $9 e-067$ \\
\hline PNAC057 & POPTR_0015s00520.1 & AT1G71930.1 & ANAC030, VND7 & 71 & $1 e-012$ \\
\hline PNAC058 & POPTR_0013s11740.1 & AT1G71930.1 & ANAC030, VND7 & 305 & $2 e-083$ \\
\hline PNAC059 & POPTR_0005s27720.1 & AT1G76420.1 & ANAC031, CUC3 & 282 & $2 e-076$ \\
\hline PNAC060 & POPTR_0002s00730.1 & AT1G76420.1 & ANAC031, CUC3 & 319 & $2 e-087$ \\
\hline PNAC061 & POPTR_0001s22770.1 & AT1G77450.1 & ANAC032 & 65 & $8 e-011$ \\
\hline PNAC062 & POPTR_0008s07950.1 & AT1G79580.3 & ANAC033 & 333 & $1 \mathrm{e}-091$ \\
\hline PNAC063 & POPTR_0010s18420.1 & AT1G79580.3 & ANAC033 & 344 & $5 e-095$ \\
\hline PNAC064 & POPTR_0019s09130.1 & AT1G79580.3 & ANAC033 & 56 & $2 e-008$ \\
\hline PNAC065 & POPTR_0003s03670.1 & AT2G02450.2 & ANAC034, ANAC035 & 318 & $2 \mathrm{e}-087$ \\
\hline PNAC066 & POPTR_0004s23970.1 & AT2G02450.2 & ANAC034, ANAC035 & 310 & $1 e-084$ \\
\hline PNAC067 & POPTR_0005s10610.1 & AT2G17040.1 & ANAC036 & 249 & $1 e-066$ \\
\hline PNAC068 & POPTR_0004s18860.1 & AT2G17040.1 & ANAC036 & 321 & 4e-088 \\
\hline PNAC069 & POPTR_0009s14370.1 & AT2G17040.1 & ANAC036 & 315 & $2 e-086$ \\
\hline PNAC070 & POPTR_0007s13910.1 & AT2G18060.1 & ANAC037, VND1 & 428 & $1 e-120$ \\
\hline PNAC071 & POPTR_0007s13910.1 & AT2G18060.1 & ANAC037, VND1 & 432 & $1 e-121$ \\
\hline PNAC072 & POPTR_0006s29180.1 & AT2G24430.2 & ANAC038, ANAC039 & 348 & $3 e-096$ \\
\hline PNAC073 & POPTR_0018s03910.1 & AT2G24430.2 & ANAC038, ANAC039 & 289 & $2 e-078$ \\
\hline PNAC074 & POPTR_0016s02780.1 & AT2G24430.2 & ANAC038, ANAC039 & 65 & $4 e-011$ \\
\hline PNAC075 & POPTR_0097s00280.1 & AT2G24430.2 & ANAC038, ANAC039 & 65 & $7 e-011$ \\
\hline PNAC076 & POPTR_0009s16290.1 & AT2G27300.1 & ANAC040, NTL8 & 272 & $2 e-073$ \\
\hline PNAC077 & POPTR_0015s00940.1 & AT2G33480.1 & ANAC041 & 100 & $9 e-022$ \\
\hline PNAC078 & POPTR_0017s06000.1 & AT2G33480.1 & ANAC041 & 89 & $3 e-018$ \\
\hline PNAC079 & POPTR_0009s07640.1 & AT2G33480.1 & ANAC041 & 59 & $3 e-009$ \\
\hline PNAC080 & POPTR_0001s11660.1 & AT2G43000.1 & ANAC042 & 245 & $2 e-065$ \\
\hline PNAC081 & POPTR_0005s22710.1 & AT2G43000.1 & ANAC042 & 268 & $2 e-072$ \\
\hline PNAC082 & POPTR_0003s14950.1 & AT2G43000.1 & ANAC042 & 245 & $3 e-065$ \\
\hline PNAC083 & POPTR_0002s05820.1 & AT2G43000.1 & ANAC042 & 296 & $7 e-081$ \\
\hline PNAC084 & POPTR_0014s10060.1 & AT2G46770.1 & ANAC043, NST1 & 381 & $1 e-106$ \\
\hline PNAC085 & POPTR_0002s17950.1 & AT2G46770.1 & ANAC043, NST1 & 370 & $1 e-103$ \\
\hline PNAC086 & POPTR_0011s15640.1 & AT2G46770.1 & ANAC043, NST1 & 298 & $6 e-081$ \\
\hline PNAC087 & POPTR_0001s35490.1 & AT3G01600.1 & ANAC044 & 259 & $2 e-069$ \\
\hline PNAC088 & POPTR_0019s13130.1 & AT3G03200.1 & ANAC045 & 87 & $3 e-018$ \\
\hline PNAC089 & POPTR_0001s26380.1 & AT3G04070.2 & ANAC047 & 189 & $3 e-048$ \\
\hline PNAC090 & POPTR_0013s05080.1 & AT3G04070.1 & ANAC047 & 338 & 4e-093 \\
\hline PNAC091 & POPTR_0019s04690.1 & AT3G04070.1 & ANAC047 & 332 & 2e-091 \\
\hline PNAC092 & POPTR_0017s05970.1 & AT3G04070.2 & ANAC047 & 87 & $8 e-018$ \\
\hline PNAC093 & POPTR_0006s04990.1 & AT3G04070.2 & ANAC047 & 122 & $3 e-028$ \\
\hline PNAC094 & POPTR_0010s23630.1 & AT3G10480.1 & ANAC050 & 418 & $1 e-117$ \\
\hline PNAC095 & POPTR_0006s02930.1 & AT3G10480.2 & ANAC050 & 75 & $5 e-014$ \\
\hline PNAC096 & POPTR_0097s00260.1 & AT3G10480.2 & ANAC050 & 70 & $1 e-012$ \\
\hline PNAC097 & POPTR_0302s00220.1 & AT3G10480.2 & ANAC050 & 74 & $2 e-013$ \\
\hline PNAC098 & POPTR_0013s07720.1 & AT3G10480.2 & ANAC050 & 64 & $6 e-011$ \\
\hline PNAC099 & POPTR_0006s02910.1 & AT3G10480.2 & ANAC050 & 74 & $2 e-013$ \\
\hline PNAC100 & POPTR_0011s12420.1 & AT3G15510.1 & ANAC056, AtNAC2 & 322 & $2 e-088$ \\
\hline PNAC101 & POPTR_0001s41490.1 & AT3G15510.1 & ANAC056, AtNAC2 & 345 & 4e-095 \\
\hline PNAC102 & POPTR_0011s05760.1 & AT3G15510.1 & ANAC056, AtNAC2 & 221 & $1 e-058$ \\
\hline PNAC103 & POPTR_0015s05450.1 & AT3G17730.1 & ANAC057 & 372 & $1 e-103$ \\
\hline
\end{tabular}


Table 1: NAC gene family in Populus (Continued)

\begin{tabular}{|c|c|c|c|c|c|}
\hline PNAC104 & POPTR_0012s03510.1 & AT3G17730.1 & ANAC057 & 350 & $4 \mathrm{e}-097$ \\
\hline PNAC105 & POPTR_0015s03700.1 & AT3G18400.1 & ANAC058 & 325 & 3e-089 \\
\hline PNAC106 & POPTR_0012s05280.1 & AT3G18400.1 & ANAC058 & 315 & $4 \mathrm{e}-086$ \\
\hline PNAC107 & POPTR_0009s02430.1 & AT3G44350.2 & ANAC061 & 256 & $8 e-069$ \\
\hline PNAC108 & POPTR_0001s22630.1 & AT3G44350.2 & ANAC061 & 277 & $5 e-075$ \\
\hline PNAC109 & POPTR_0006s19350.1 & AT3G44350.1 & ANAC061 & 81 & $8 e-016$ \\
\hline PNAC110 & POPTR_0015s00640.1 & AT3G49530.1 & ANAC062 & 307 & $9 e-084$ \\
\hline PNAC111 & POPTR_0012s00760.1 & AT3G49530.1 & ANAC062 & 306 & $2 e-083$ \\
\hline PNAC112 & POPTR_0013s09280.1 & AT4G10350.1 & ANAC070 & 436 & $1 e-123$ \\
\hline PNAC113 & POPTR_0019s09400.1 & AT4G10350.1 & ANAC070 & 451 & $1 e-127$ \\
\hline PNAC114 & POPTR_0003s08830.1 & AT4G17980.1 & ANAC071 & 296 & $1 e-080$ \\
\hline PNAC115 & POPTR_0001s02710.1 & AT4G17980.1 & ANAC071 & 296 & $1 \mathrm{e}-080$ \\
\hline PNAC116 & POPTR_0019s13140.1 & AT4G17980.1 & ANAC071 & 89 & $5 e-018$ \\
\hline PNAC117 & POPTR_0009s05690.1 & AT4G27410.2 & ANAC072, RD26 & 189 & $5 e-048$ \\
\hline PNAC118 & POPTR_0011s12400.1 & AT4G27410.2 & ANAC072, RD26 & 353 & $9 e-098$ \\
\hline PNAC119 & POPTR_0007s02050.1 & AT4G27410.2 & ANAC072, RD26 & 87 & $1 e-017$ \\
\hline PNAC120 & POPTR_0001s41460.1 & AT4G27410.2 & ANAC072, RD26 & 375 & $1 e-104$ \\
\hline PNAC121 & POPTR_0017s04800.1 & AT4G28500.1 & ANAC073 & 344 & 4e-095 \\
\hline PNAC122 & POPTR_0007s01350.1 & AT4G28500.1 & ANAC073 & 337 & $6 e-093$ \\
\hline PNAC123 & POPTR_0011s05740.1 & AT4G28500.1 & ANAC073 & 345 & 2e-095 \\
\hline PNAC124 & POPTR_0004s04900.1 & AT4G28500.1 & ANAC073 & 355 & 2e-098 \\
\hline PNAC125 & POPTR_0005s24750.1 & AT4G28530.1 & ANAC074 & 271 & $4 e-073$ \\
\hline PNAC126 & POPTR_0002s03830.1 & AT4G28530.1 & ANAC074 & 276 & $7 e-075$ \\
\hline PNAC127 & POPTR_0006s 15740.1 & AT4G29230.1 & ANAC075 & 511 & $1 e-145$ \\
\hline PNAC128 & POPTR_0006s15740.1 & AT4G29230.1 & ANAC075 & 486 & $1 e-137$ \\
\hline PNAC129 & POPTR_0010s23650.1 & AT5G04410.1 & ANAC078, NAC2 & 483 & $1 e-136$ \\
\hline PNAC130 & POPTR_0008s03170.1 & AT5G04410.1 & ANAC078, NAC2 & 499 & $1 e-141$ \\
\hline PNAC131 & POPTR_0007s03840.1 & AT5G09330.3 & ANAC082 & 265 & $4 e-071$ \\
\hline PNAC132 & POPTR_0005s06040.1 & AT5G09330.3 & ANAC082 & 256 & $2 e-068$ \\
\hline PNAC133 & POPTR_0015s11420.1 & AT5G13180.1 & ANAC083 & 238 & $2 e-063$ \\
\hline PNAC134 & POPTR_0001s33260.1 & AT5G13180.1 & ANAC083 & 162 & $1 e-040$ \\
\hline PNAC135 & POPTR_0017s09030.1 & AT5G13180.1 & ANAC083 & 151 & $3 e-037$ \\
\hline PNAC136 & POPTR_0003s16490.1 & AT5G13180.1 & ANAC083 & 310 & 4e-085 \\
\hline PNAC137 & POPTR_0001s13380.1 & AT5G13180.1 & ANAC083 & 309 & $1 e-084$ \\
\hline PNAC138 & POPTR_0012s10530.1 & AT5G13180.1 & ANAC083 & 228 & $3 e-060$ \\
\hline PNAC139 & POPTR_0004s11840.1 & AT5G17260.1 & ANAC086 & 89 & 7e-019 \\
\hline PNAC140 & POPTR_0012s03100.1 & AT5G17260.1 & ANAC086 & 87 & $4 e-018$ \\
\hline PNAC141 & POPTR_0012s03130.1 & AT5G17260.1 & ANAC086 & 95 & $1 e-020$ \\
\hline PNAC142 & POPTR_0014s10370.1 & AT5G17260.1 & ANAC086 & 139 & $2 e-033$ \\
\hline PNAC143 & POPTR_0012s03120.1 & AT5G17260.1 & ANAC086 & 68 & $6 e-019$ \\
\hline PNAC144 & POPTR_0019s04710.1 & AT5G18270.2 & ANAC087 & 267 & $1 e-071$ \\
\hline PNAC145 & POPTR_0013s05110.1 & AT5G18270.2 & ANAC087 & 248 & $6 e-066$ \\
\hline PNAC146 & POPTR_0016s07680.1 & AT5G22380.1 & ANAC090 & 218 & $3 e-057$ \\
\hline PNAC147 & POPTR_0016s07690.1 & AT5G22380.1 & ANAC090 & 213 & $7 e-056$ \\
\hline PNAC148 & POPTR_0006s22580.1 & AT5G22380.1 & ANAC090 & 242 & $2 e-064$ \\
\hline PNAC149 & POPTR_0097s00210.1 & AT5G24590.2 & ANAC091, TIP & 56 & $3 e-008$ \\
\hline PNAC150 & POPTR_0017s08490.1 & AT5G39820.1 & ANAC094 & 50 & $3 e-006$ \\
\hline PNAC151 & POPTR_0011s11600.1 & AT5G53950.1 & ANAC098, CUC2 & 311 & $3 e-085$ \\
\hline PNAC152 & POPTR_0001s40680.1 & AT5G53950.1 & ANAC098, CUC2 & 300 & $1 e-081$ \\
\hline PNAC153 & POPTR_0017s12210.1 & AT5G61430.1 & ANAC100, ATNAC5 & 377 & $1 e-105$ \\
\hline PNAC154 & POPTR_0015s02170.1 & AT5G61430.1 & ANAC100, ATNAC5 & 446 & $1 e-125$ \\
\hline PNAC155 & POPTR_0012s01610.1 & AT5G61430.1 & ANAC100, ATNAC5 & 448 & $1 e-126$ \\
\hline
\end{tabular}


Table 1: NAC gene family in Populus (Continued)

\begin{tabular}{|c|c|c|c|c|c|}
\hline PNAC156 & POPTR_0006s24740.1 & AT5G62380.1 & ANAC101, VND6 & 61 & $1 e-009$ \\
\hline PNAC157 & POPTR_0004s10720.1 & AT5G62380.1 & ANAC101, VND6 & 74 & $9 e-014$ \\
\hline PNAC158 & POPTR_0005s08440.1 & AT5G62380.1 & ANAC101, VND6 & 74 & $2 e-013$ \\
\hline PNAC159 & POPTR_0014s16160.1 & AT5G62380.1 & ANAC101, VND6 & 59 & $6 e-009$ \\
\hline PNAC160 & POPTR_0007s04250.1 & AT5G64530.1 & ANAC104, XND1 & 185 & $2 e-047$ \\
\hline PNAC161 & POPTR_0003s01720.1 & AT5G64530.1 & ANAC104, XND1 & 231 & $2 e-061$ \\
\hline PNAC162 & POPTR_0001s21440.1 & AT5G64530.1 & ANAC104, XND1 & 215 & 1e-056 \\
\hline PNAC163 & POPTR_0005s06510.1 & AT5G64530.1 & ANAC104, XND1 & 199 & $1 e-051$ \\
\hline
\end{tabular}

Gene loci are from the Phytozome website http://www.phytozome.net/, release 2.0). A complete list of the coding sequences (CDS), deduced amino acid sequences and genomic DNA sequences is available in Additional file 1.

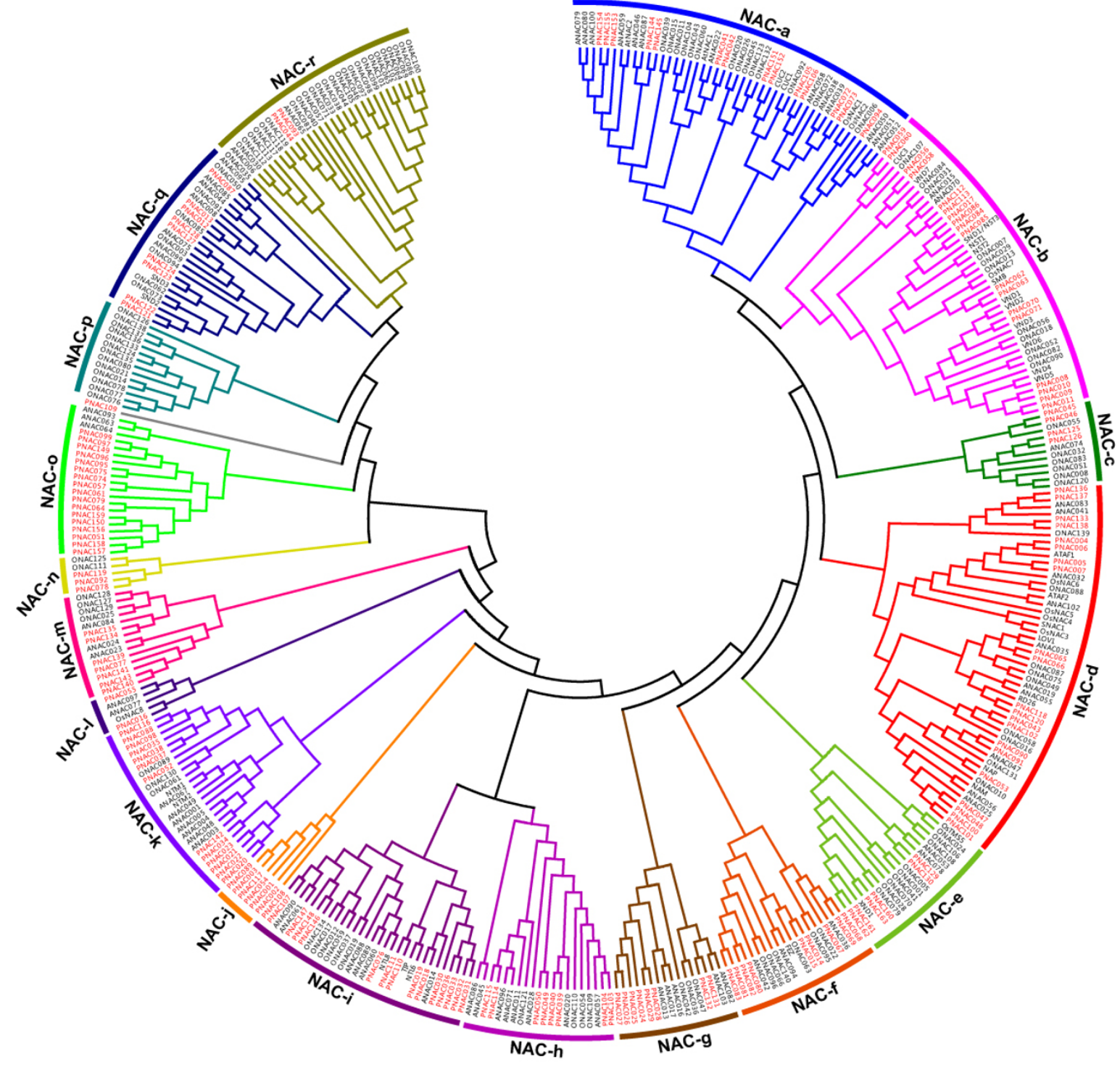

Figure 1 Joined phylogenetic tree of NAC domain-containing proteins from Populus, Arabidopsis and rice. The deduced full-length amino acid sequences of 163 Populus, 105 Arabidopsis and 140 rice NAC genes were aligned by Clustal X 1.83 and the phylogenetic tree was constructed using MEGA 4.0 by the Neighbor-Joining (NJ) method with 1,000 bootstrap replicates. Each NAC subfamily was indicated in a specific color. Members of NAC protein from Populus were denoted in red. 
that the expansion of NAC genes occurred before the divergence of Populus, Arabidopsis and rice. Noticeably, NAC genes with same functions showed a tendency to fall into one subgroup, similar to the previous reports $[7,8,57]$. For instances, subfamily NAC-a encompassed the NAC proteins involved in shoot organ boundary delimitation (eg. CUC1, 2) [25,26], whereas subfamily NAC-d possessed all the NAC proteins that function in shoot apical meristem (SAM) establishment, pattern formation in embryos and flowers (eg. NAM) [11], stress responses (eg. ATAF1, RD26 and OsNAC6) $[18,47,66]$ and leaf senescence (eg. NAP) [29]. This group also encompassed ANAC019 and ANAC055, which have been shown to be induced by abiotic stresses (ABA, drought and salinity) and to enhance tolerance to drought when ectopically over-expressed [40]. The membrane-associated NAC proteins that mediate either cytokinin signaling during cell division or endoplasmic reticulum stress responses were clustered into the NAC$\mathrm{i}$ and NAC-k subfamilies. The members in these two subgroups are distinct with other NACs in that they harbor a transmembrane (TM) motif with a predicted $\alpha$-helix in the far C-terminal region. In NAC-k subfamily, there were only two well functionally characterized members namely NTM1 and NTM2. The well-characterized genes in subfamily NAC-f included FEZ, which was demonstrated to be associated with the orientation of cell division in root stem cells [34]

Remarkably, subfamily NAC-j did not include any Arabidopsis and rice NAC proteins but only members from Populus, suggesting that they may have been either lost in Arabidopsis and rice or acquired in the Populus lineages after divergence from the last common ancestor. We speculate that these subsets of genes may also have specialized roles with respect to the woody perennial habit in Populus. Phylogenetic analysis also revealed a subgroup NAC-o that contained sequence representatives in Populus and Arabidopsis but not in rice, indicating that the NAC members in this subgroup were acquired or differentially retained in eudicots post-divergence from monocots. In contrast, the subgroup NAC-p was composed of 13 rice NAC proteins, but no Arabidopsis and Populus proteins, which suggests diversification and expansion of this subgroup after the monocot-eudicot radiation.

The NAC proteins associated with secondary wall formation in fiber and vascular vessel development were divided into three independent subfamilies. All NSTs (NST1, NST2, NST3/SND1) and VNDs (VND1-VND7) were clustered into one subfamily NAC-b, whereas SND2, SND3 and their counterparts were grouped into NAC-q. The other well-characterized NAC member XND1 in secondary wall formation was assigned to another different subfamily (NAC-e). All the six functional characterized NST3/SND1 orthologs (PtWND1B to 6B) in Populus fell into subfamily NAC-b [19]. Although enormous evidences exemplified that all these NAC genes performed as key transcriptional switches in the secondary cell wall formation process, their exact functional roles diversified [52-55]. The phylogenetic analysis conducted herein may provide a potential supporting for their functional diversities. What's more, the tissue-specific expression profiling available on GENEVESTIGATOR lent further supports for this notion [67]. The expression pattern of XND1 in Arabidopsis was extremely different from that of NSTs and VNDs. Nevertheless, the expression pattern of SND2 and SND3 was somewhat more similar to that of SND1/NST3 and NST2 (Additional file 4). In accordance with the recent report, NST, VND, SND, and XND from 11 different species were also classified into three distinct phylogenetic subgroups conducted by Maximum Likelihood (ML) algorithm [57]. Another well-characterized gene namely SOMBRERO (SMB), which has been shown to control the reorientation and timing of cell division in root stem cells by negatively regulating FEZ activity [34], was clustered together with NSTs and VNDs into subfamily NAC-b. We inspected the expression patterns of SMB on GENESTIGATOR [67] but failed in an attempt to find any clues for their diversified roles in secondary wall formation. SMB was specifically highly expressed in callus and lateral root cap rather than secondary cell wall enriching tissues, suggesting a specific role in cell division and root development (Additional file 4).

It is noteworthy that the number of Populus genes was generally overrepresented than that of Arabidopsis and rice in almost all clades, especially within NAC-o subfamily, in which Populus NAC genes was particularly overrepresented and almost sixfold the number of genes with respect to Arabidopsis. Alternatively, in subfamily NAC-a and q, the number of Arabidopsis and rice genes nearly equaled that of Populus. In contrast, the number of rice NAC genes in subfamily NAC-r showed an overwhelming predominance with respect to that of Populus.

The phylogenetic tree obtained in this study is largely consistent with previous analyses [6-8,57], although there are some discrepancies. The first systematic analysis of Arabidopsis and rice NAC proteins classified them into 18 subgroups [6]. However, another phylogenetic analysis of rice NAC proteins suggested that the NAC family can be divided into five groups and each subfamily was largely diversified [7]. In a report concerning soybean NAC family, the genes were clustered into 15 distinct subfamilies with robust bootstrap supporting [8]. More recently, systematic phylogenetic analyses of numerous NAC proteins from a wide range of plant species indicated that NAC proteins can be divided into 
eight subfamilies [57]. In our opinions, the main reason for the discrepancies of the phylogenetic trees reported may lie in the fact that all the previous NAC protein classifications were based on the conserved $\mathrm{N}$-terminal NAC domains, either from sub-domain A to D or A to $\mathrm{E}$, which did not take the highly divergent $\mathrm{C}$-terminal sequences into consideration [6-8,57]. In an attempt to gain better understanding of the phylogeny of NAC gene family, we performed the phylogenetic analysis with inclusion of the highly diverse C-terminal sequence. Moreover, different algorithms exploited in the phylogenetic analyses may lead to the inconsistent interpretations. In the previous analyses, different algorithms including Neighbor-Joining (NJ) [6,8], Maximum likelihood (ML) [57] and Bayesian method [7] were implemented, which may make the results less comparable.

Inspection of the phylogenetic tree topology revealed several pairs of NAC proteins with a high degree of homology in the terminal nodes of each subfamily, suggesting that they are putative paralogous pairs (homologous genes within a species that diverged by gene duplication) (Figure 1 and Additional file 3). Totally, forty-nine pairs of putative paralogous NAC proteins were identified, accounting for more than $60 \%$ of the entire family, with sequence identities ranging from $71 \%$ to $97 \%$ (see Additional file 5 for details).

\section{Chromosomal location and gene duplication of Populus NAC genes}

In silico mapping of the gene loci showed that 163 Populus NAC genes were distributed across all 19 Linkage Groups (LG). In the currently released sequences, totally 120 NAC genes were mapped to LGs, whereas 43 genes were remained on as yet unmapped scaffolds. The distributions of Populus NAC genes across the LGs appeared to be non-random (Figure 2). LG II encompassed the largest number of 12 NAC genes followed by 10 on LG V and LG I, respectively. In contrast, only one NAC gene was found on LG XVIII and two NAC genes were on LG XVII. Substantial clustering of Populus NAC genes was evident on several LGs, especially on those with high densities of NAC genes. For instances, PNAC020, 022 and 030 were cluster localized on a 17 kb segment on LG XIV, and three NACs (PNAC033, 034 and 035) were organized in another cluster within a $10 \mathrm{~kb}$ fragment on LG II, whereas PNAC140, 141 and 143 were arranged in a cluster localized to a $11 \mathrm{~kb}$ segment on LG XII (Figure 2).

Previous studies revealed that Populus genome had undergone at least three rounds of genome-wide duplications followed by multiple segmental duplication, tandem duplication, and transposition events such as retroposition and replicative transposition $[5,68]$.
Among them, the segmental duplication associated with the salicoid duplication event that occurred 65 million years $(\mathrm{Ma})$ ago significantly contribute to the amplification of many multi-gene families [61,69-71]. To determine the possible relationship between the NAC genes and potential segmental duplications, we mapped Populus NACs to the duplicated blocks established in the previous studies [5]. The distributions of NAC genes relative to the corresponding duplicate genomic blocks were illustrated in Figure 2. Within the 36 identified duplicated blocks associated with the recent salicoid duplication event, about 73\% (87 of 120) of Populus NACs were preferentially retained duplicates that locate in both duplicated regions of 28 block pairs, whereas nine block pairs only harbored NACs on one of the blocks and lack the corresponding duplicates, suggesting that dynamic changes may have occurred following segmental duplication, leading to loss of some of the genes. In contrast, only a small number of 19 NAC genes were located outside of any duplicated blocks.

In addition, the tandem duplications might also have an impact on the expansion of the NAC gene family. Fine mapping analysis revealed the presence of 13 pairs of adjacent genes within a $20 \mathrm{~kb}$ distance possibly due to tandem duplication either in inverse or same orientation (Figure 2). It is intriguing that except for one pair (PNAC110 and 119), all the other putative tandem duplicates were represented within the recent segmental duplicate blocks. Similar results have also been reported in several other Populus gene families [71,72]. These 26 NAC genes (16\%) were represented in 12 distinct tandem duplicate gene clusters, with ten clusters containing two tandem genes and two clusters possessing three tandem genes. But further analysis of their similarities demonstrated that most of the tandem clustered NAC pairs shared relative low similarities (mostly below 50\%), which may exclude them from tandem duplicate candidates. Therefore, ultimately only six pairs of NACs were conformed to the standards as tandem duplicates, which were represented in tandem clusters of two to three genes each. These tandem duplicated NAC genes were organized onto duplicated blocks, implying that the occurrence of local duplications was prior to the chromosomal segment duplication.

As a considerable proportion of the NAC proteins appeared to be paralogous pairs as revealed in the phylogenetic analysis, we further investigated whether traceable genome duplication events have contributed to the expansion of the NAC family. Nineteen out of 49 homologous pairs remained in conserved positions on segmental duplicated blocks (Figure 2), suggesting that these genes may be the result of segmental duplication event during the evolution. For 26 homologous pairs, no traceable duplication events could be inferred, because 


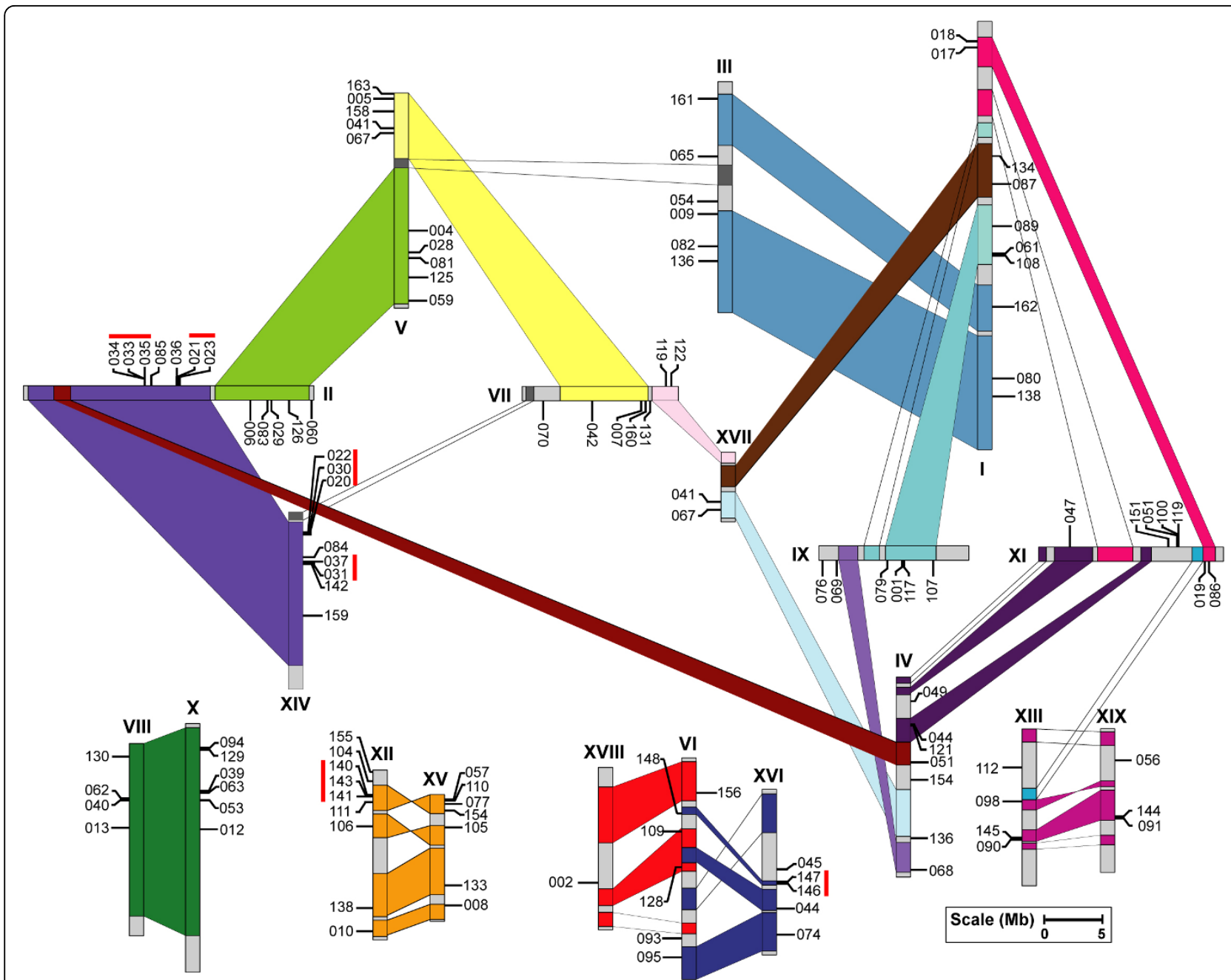

Figure 2 Chromosomal locations of Populus NAC genes. Only 120 NAC genes are mapped to the 19 Linkage Groups (LG), while the other 43 genes reside on unassembled scaffolds. The schematic diagram of genome-wide chromosome organization arisen from the salicoid genome duplication event in Populus was adapted from Tuskan et al., (2006) [5]. Segmental duplicated homeologous blocks are indicated with the same color. Only the duplicated regions containing NAC genes are connected with lines in shaded colors. Tandemly duplicated genes are indicated with red lines. Scale represents a $5 \mathrm{Mb}$ chromosomal distance.

they are mapped to the yet non-assembled scaffolds. Among them, a total of 11 genes were located on segmental duplication blocks with their counterparts not mapped to LGs yet. Meanwhile, a subset of eight NAC genes were located outside of the segmental duplication blocks while their corresponding members mapped to non-assembled scaffolds. Taken together, nine genes of the homologous pairs were definitely not located on any duplicated blocks, and only three of them had the homologous counterparts located on the duplicated blocks. Duplicates of these genes appeared to have lost from the Populus genome. Interestingly, one homologous pair PNAC121 and 122 was located on two divergent rather than homologous duplicated blocks, thus we could not figure out any traceable duplication history for this pair of NACs even though they were covered by duplicated blocks.

Based primarily on the genomic organization of NAC genes, we could conclude that segmental duplications exclusively contributed to the complexity of NAC gene family in the Populus genome. Similarly, segmental duplications have also been shown to contribute to the expansion of other gene families in Populus [61,70-72]. Our studies indicated that Populus NAC genes have been preferentially retained at a rate of $73 \%$, which is much higher than the average rate following the salicoid genome-wide duplication and rearrangement events [5]. On a genome-wide scale, approximately one third of predicted genes are retained in duplications resulting from the salicoid duplication event [5]. The high 
retention rate of duplicated genes was also previously reported in other Populus gene families [70,71]. These findings are also in consistency with the previous studies demonstrating that genes involved in transcription regulation and signal transduction are preferentially retained [73-75]. Another plausible explanation to the relatively high retention rate of duplicate genes in NAC gene family may lie in the fact that Populus genome has been indicated to evolve at a much slower rate compared to that of Arabidopsis [5]. The duplicated genes may undergo divergent fates during subsequent evolution such as nonfunctionalization (loss of original functions), neofunctionalization (acquisition of novel functions), or subfunctionalization (partition of original functions) [76,77]. Whether the duplicated NAC genes correspond to genetic redundancy or have evolved divergent functions remains to be further functionally characterized.

The tandem duplication ratio of NAC genes in this study is relatively lower compared to some other gene families in Populus, which was represented in a considerable higher proportion [71,72]. Interestingly, a similar low retention rate of tandem duplicates was also observed for Populus ARF gene family [71].

\section{Gene structure and conserved motifs of Populus NAC genes}

It is well known that gene structural diversity is a possible mechanism for the evolution of multigene families. In order to gain further insights into the structural diversity of NAC genes, we compared the exon/intron organization in the coding sequences of individual NAC genes in Populus. A detailed illustration of the exon/ intron structures was shown in Figure 3B. In addition, a separate phylogenetic tree was generated from complete protein sequences of all the NAC genes in Populus, which divided the NAC genes into ten subfamilies (Figure $3 \mathrm{~A}$ ). In general, most closely related members in same subfamilies shared similar exon/intron structure in terms of intron number and exon length (Figure 3B). For instances, the NAC genes in subfamily I, III, IV and VII contained two to three introns while those in subfamily IX all possessed no introns with exception of PNAC079, which harbored one intron. In contrast, the gene structure appeared to be more variable in subfamilies II and V, which had the largest number of exon/ intron structure variants with striking distinctions. Interestingly, although the exon/intron organization of NACs varied significantly in terms of intron number, the intron phase was remarkably high conserved (Additional file 6), which was indicative of exon shuffling during the evolution process [78].

We further analyzed the exon/intron structure of the paralogous pairs in NAC gene family to obtain traceable intron gain or loss information about these genes. Of the 49 paralogous pairs, thirty-four pairs showed conserved exon/intron structure either in terms of intron numbers or gene length (Figure 3B). Remarkably, twenty-five pairs of genes exhibited a highly conserved distribution of exons and introns, with all members having three exons and two introns. Despite a majority of these genes have arisen from segmental duplication events, the others were located either on non-duplicated blocks or on not-yet assembled scaffolds. While the gene structure was conserved between some paralogous pairs, others exhibited some extent of variation. For example, eleven NAC genes out of the 49 paralogous pairs possessed three exons in their coding regions, whereas their homologous counterparts all contained four exons within nearly identical gene lengths (Figure $3 \mathrm{~B})$. We could firmly speculate that the differences in the last exons are probably derived from single intron loss or gain occurred during the process of structural evolution of NAC paralogs. The conserved exon/intron architecture shared by these homologous gene pairs may reciprocally lend supports for the results from the phylogenetic analysis and the duplication events.

To further reveal the diversification of NAC genes in Populus, putative motifs were predicted by the program MEME (Multiple Expectation Maximization for Motif Elicitation), and 20 distinct motifs were identified. As expected, most of the closely related members in the phylogenetic tree had common motif compositions, suggesting functional similarities among the NAC proteins within the same subfamily (Figure $3 \mathrm{C}$ ). However, the biological significance of most of the putative motifs remains to be elucidated as they do not have homologs when searching against Pfam and SMART (Simple Modular Architecture Research Tool) databases $[79,80]$. The details of the 20 putative motifs were referred in Additional file 7. As illustrated in the previous studies, most of the NAC proteins possessed A to $E$ subdomains in the $\mathrm{N}$ termini that conferred the DNA-binding activities. In this study, motif 2, 5, 1, 3 and 6 specifying the NAM subdomain A to E respectively were present in most of the NAC family members in Populus, whereas a small subset of sequences did not have all five motifs in the typical NAC DNA-binding domains. Even though the C-terminal regions of NACs were highly divergent, we could also identify at least eight conserved motifs using the MEME motif search tool. Noticeably, some specific motifs were present in NACs from specific subfamilies, for instance, motif 16 for subfamily $\mathrm{V}$, motif 9 for subfamily VIII and motif 11 for subfamily IX. Whether these motifs confer unique functional roles to NACs remains to be further investigated.

Of the 49 paralogous pairs, thirty-seven pairs of NACs shared conserved motif composition with each other, indicative of functional similarities. In contrast, specific 


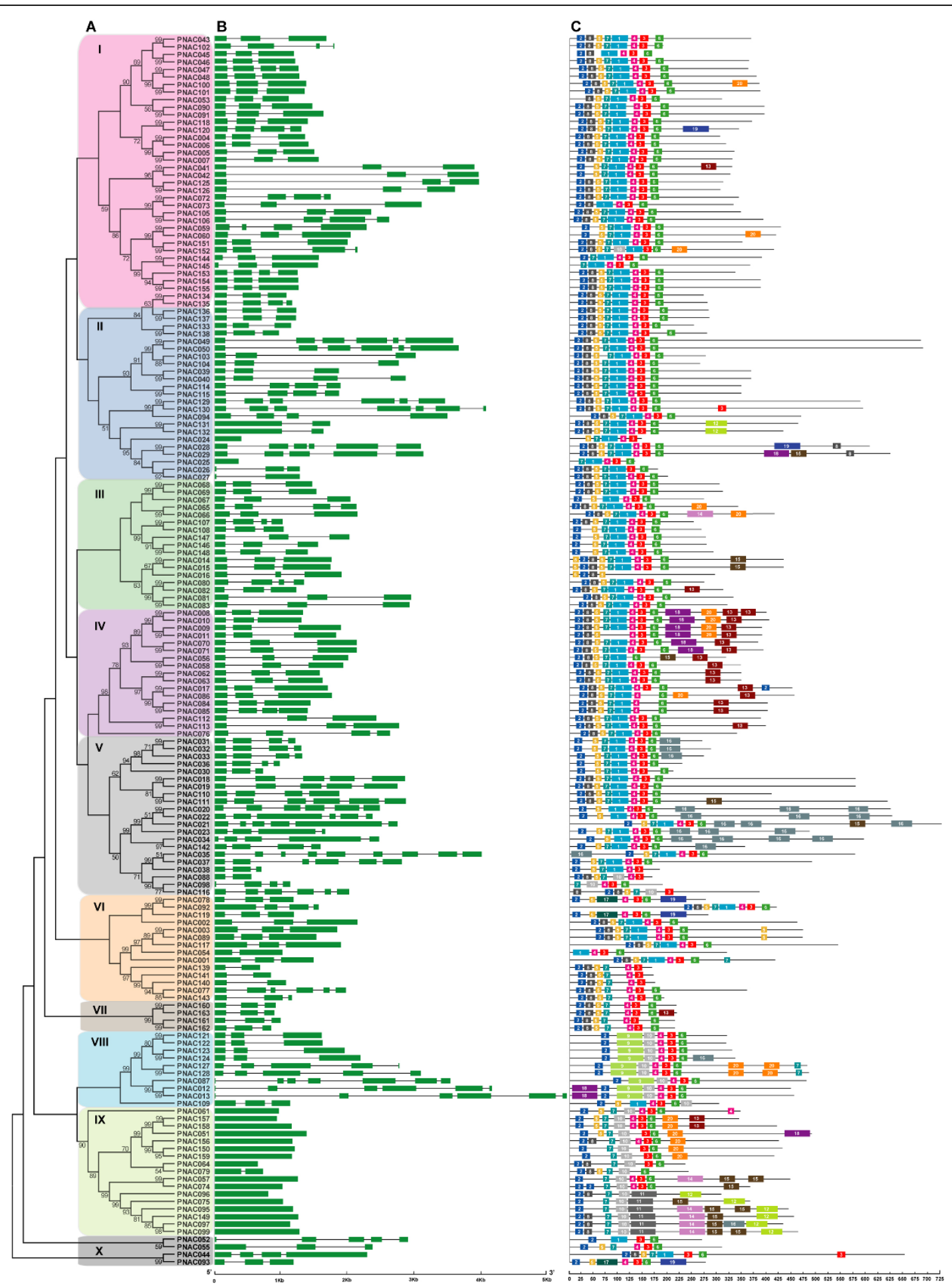

Figure 3 Phylogenetic relationships, gene structure and motif compositons of Populus NAC genes. A. Multiple alignments of 163 fulllength amino acids of NAC genes from Populus were executed by Clustal X 1.83 and the phylogenetic tree was constructed using MEGA 4.0 by the Neighbor-Joining (NJ) method with 1,000 bootstrap replicates. The percentage bootstrap scores higher than $50 \%$ are indicated on the nodes. The ten major phylogenetic subfamilies designated as I to X are marked with different color backgrounds. B. Exon/intron structures of NAC genes from Populus. Exons and introns are represented by green boxes and black lines, respectively. The sizes of exons and introns can be estimated using the scale at bottom. C. Schematic representation of the conserved motifs in the NAC proteins from Populus elucidated by MEME. Each motif is represented by a number in the colored box. The black lines represent the non conserved sequences. Refer to Additional file 7 for the details of individual motif. 
motifs located in the $\mathrm{C}$ termini were observed for the rest 12 pairs of NACs, for example, motif 13 for PNAC041, 082, 113, 163, motif 14 for PNAC066, and motif 19 for PNAC120. We attempt to speculate that these specific motifs may by some extent attribute to the subfunctionalization or neofunctionalization of the duplicated genes during subsequent evolution processes.

The similar gene structures and conserved motifs of NAC genes in same subfamilies may provide additional supports to the phylogenetic analysis. On the other hand, the differences between gene organization and the divergences in motif compositions among different subfamilies may also indicate that Populus NACs are functionally diversified.

\section{Differential expression profile of Populus NAC genes}

Publicly available Expressed Sequence Tags (ESTs) was considered as a useful means of studying gene expression profiles (digital northern) [81]. A scrutiny of the frequency of ESTs in different databases allows preliminary analysis of gene expression under various conditions and across tissues. The digital expression profiles of 81 Populus NAC genes were obtained from EST databases at NCBI (Oct., 2009), while the rest of other 82 NAC genes did not have corresponding EST sequences in the database. Noticeably, the frequency of ESTs was low with most of NACs represented by a single EST sequence, which is consistent with the commonly low transcript abundance feature of transcription factor genes [61]. Thus we postulate that the absent genes in EST database might either transcribe at relatively too low abundance to be detected or had special temporal and spatial expression patterns not examined in the libraries. Another possible explanation is that these genes might be pseudogenes, as they only possessed partial NAC domains as described previously (Figure 3C). In order to gain a better portrait of NAC gene expression profiles, we further largely divided the EST data into two groups, with one group contained all woodforming tissues and the other derived from the other tissues. The EST digital expression profiles were illustrated in Figure 4, Figure 5 and Additional file 8. The expression profiles demonstrated that majority NACs have rather broad expression patterns with presence in diverse libraries. Not surprisingly, a considerable subset of genes was preferentially expressed in leaves (Figure 4). In addition to leaves, more than $40 \%$ (36 of 81) of the NAC genes were especially expressed during wood formation (in cambial zone and xylem) (Figure 5).

It is noteworthy that several putative NAC orthologs between Arabidopsis and Populus showed strikingly consistent expression patterns, which lend further supporting to the existence of functional conservation between these two species. For example, the expression profiles of homologous pair PNAC031 and 032, which had two corresponding ESTs derived from shoot meristem respectively (Figure 4), further reinforced the notion that they are the closest orthologs of NAM in Arabidopsis [11]. The EST data revealed that PNAC013 gene was specifically expressed in active cambium with two detectable ESTs and highly resembled the expression pattern of its closest ortholog ANAC009 in Arabidopsis, which was expressed predominantly in highly dividing and expanding tissues such as callus and root cap (Additional file 4).

Although the digital EST expression analyses provided a first glimpse of the patterns of NAC expression in Populus, we could not draw conclusive inferences regarding NAC gene expression due to the obvious limitations of EST data such as relative narrow sample coverage and biases towards highly expressed genes. Thus, we performed a more comprehensive microarray analyses for NAC gene expression profiles. To gain more insight into the expression patterns of NAC genes, a comprehensive expression analysis was further performed by utilizing the publicly available microarray data for Populus. Firstly, we re-analyzed the collections of microarray data from wood-formation series available at PopGenIE, a database repository of transcriptomics data available for Populus $[82,83]$. Distinct expression profiles were identified for a total of 29 NAC genes (Figure 6 and Additional file 9), whereas no corresponding probe sets were available for the remaining NACs in the datasets. The relative low coverage of NACs in the datasets analyzed maybe partially due to the fact that these arrays were mainly derived from ESTs rather than offered a whole genome coverage [84].

Two subset of NAC genes exhibited specifically high transcript accumulation in active and dormant cambiums respectively, from two independent microarray datasets $[85,86]$, strongly indicating their specific roles in wood-formation (Figure 6A and 6B). In addition, by incorporating the different microarray data, we were capable to identify different subsets of NAC genes displaying specifically high expression levels in phloem, differentiating xylem and mature xylems, respectively. The expression levels of five NAC genes including PNAC070, 086, 121 and 122 were peaked exclusively in differentiating xylems supported by two independent microarray datasets (Figure 6C and 6G) $[87,88]$. Among them, PNAC070 (PtWND5A), PNAC086 (PtWND1A) and PNAC008 (PtWND3A) were demonstrated in previous studies as putative redundant homologs involved in Populus wood formation [19]. Interestingly, several genes such as PNAC013, 020 and 120 were preferentially expressed in tissues enriched of secondary cell walls, whereas a subset of genes including PNAC028, 052, 055 and 023 showed constitutively high expressions 


\begin{tabular}{|c|c|}
\hline Whoot meristem & Developing vegetative buds \\
\hline Werminal vegatative buds & Catkin \\
\hline afloral bud & DFlower \\
\hline Imbibed seed & Juvenile leaf \\
\hline$\square$ ¿Senescing leaf & aLeaf \\
\hline apetiole & $\square$ stem \\
\hline Eark & $\because$ Root \\
\hline
\end{tabular}

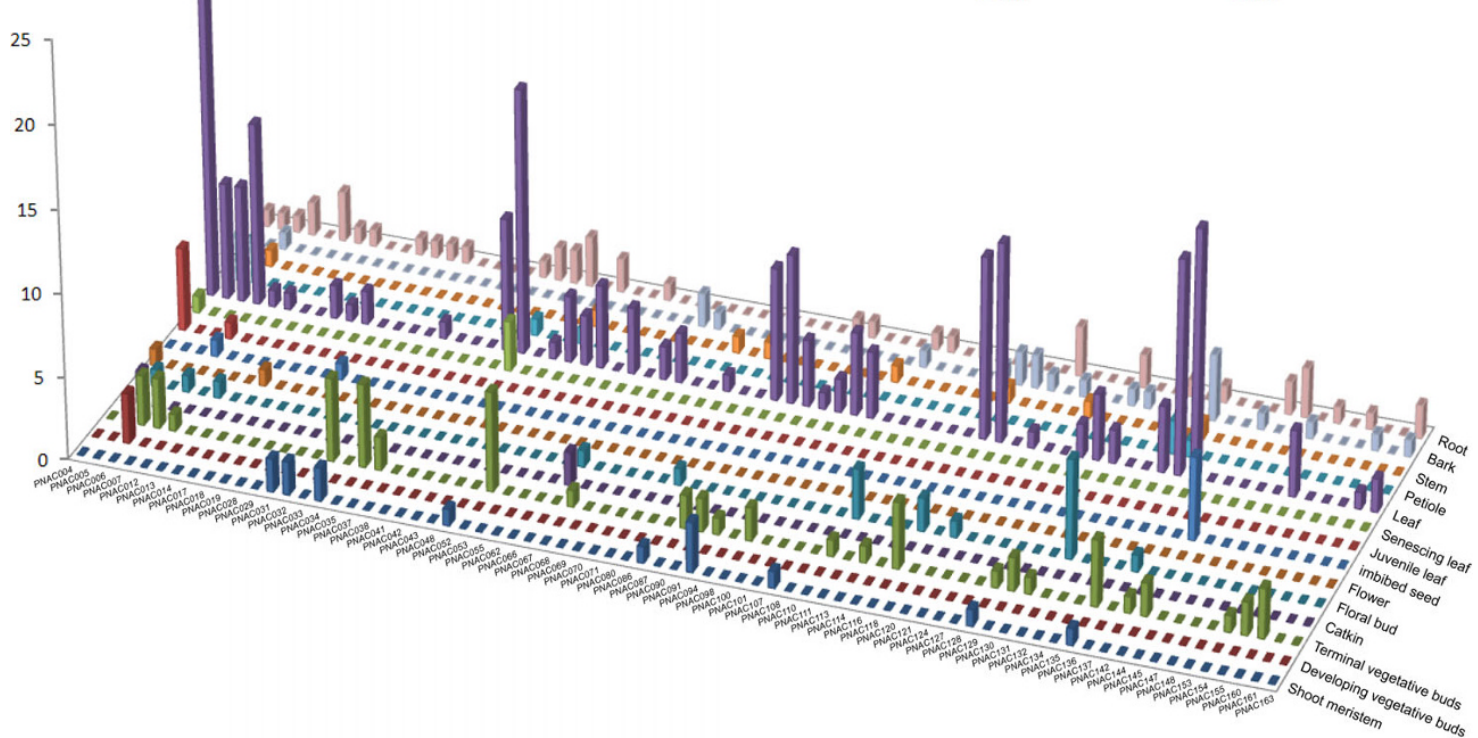

Figure 4 In silico EST analysis of Populus NAC genes. EST frequency of 74 Populus NAC genes was obtained by screening the EST datasets from various libraries across a set of 14 tissues. Expression of NAC genes was plotted as counts of corresponding ESTs for particular gene in the database.

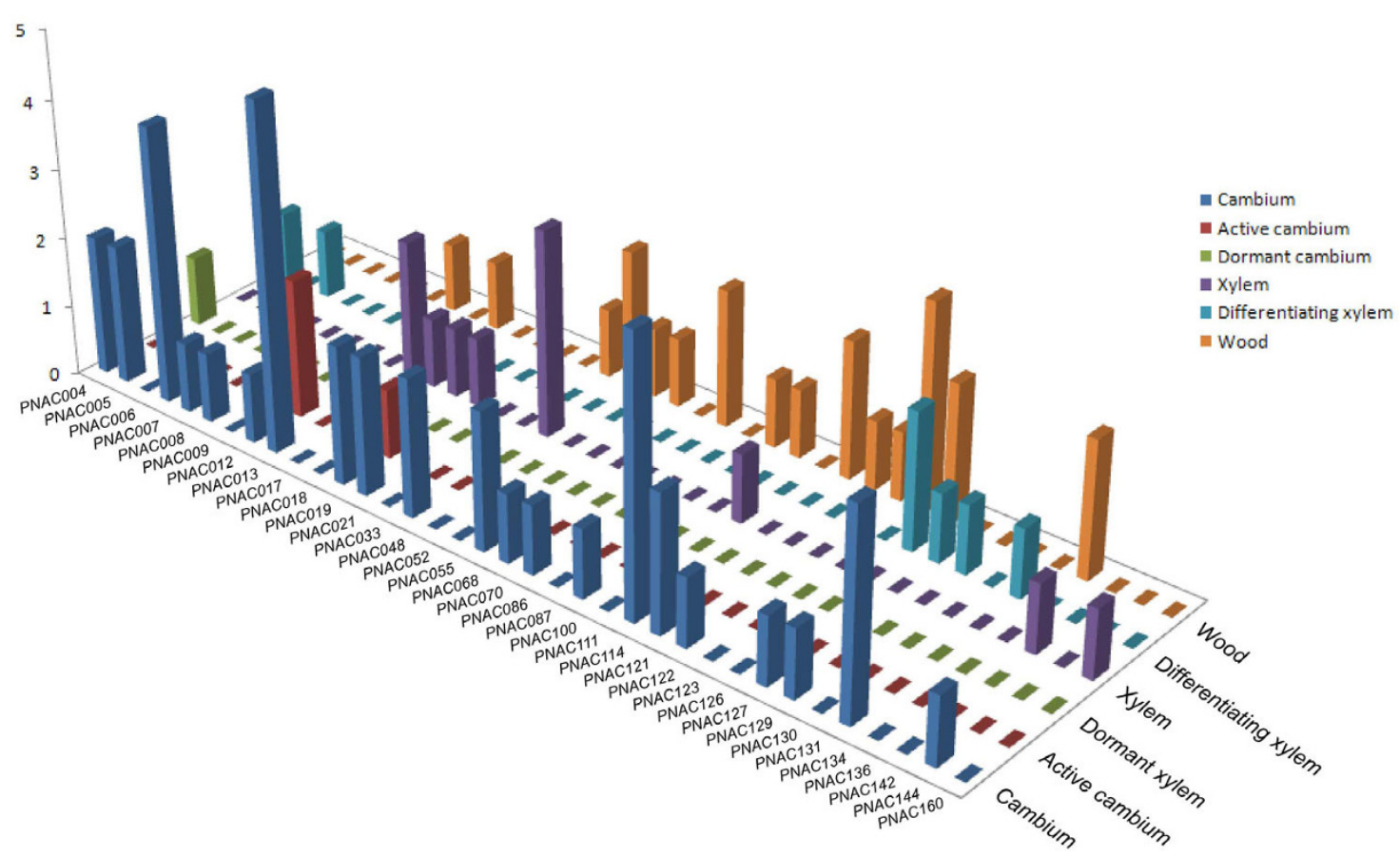

Figure 5 EST profiles of Populus NAC genes in wood-forming tissues. EST distribution of 36 Populus NAC genes was obtained by searching the EST datasets originated from libraries of wood-forming tissues. Expression of NAC genes was plotted as counts of corresponding ESTs for particular gene in the database. 


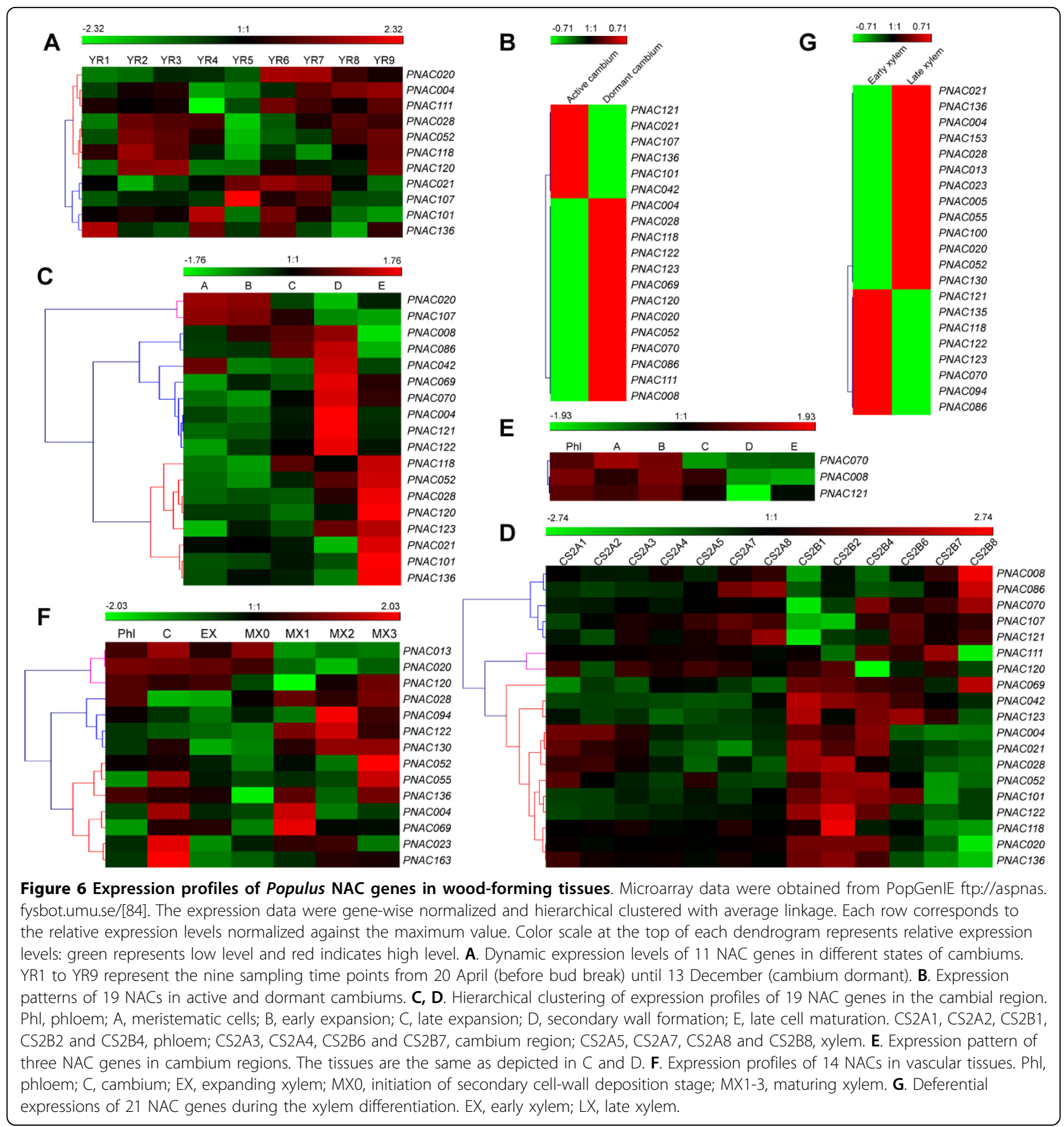

in both secondary and primary cell wall enriching tissues (Figure 6A-E). Similarly, several genes such as PNAC118 and 136 displayed varied peak abundance of transcript across the same tissues tested among different microarray datasets (Figure 6A-E).

As the previous analyzed microarray are mainly derived from ESTs and only a small fraction of NACs is present in these datasets, we further seek to use microarray based on whole genome coverage to provide deep insights into the expression patterns of NACs. We have reanalyzed the Populus microarray data generated by Wilkins and coworkers [61]. Only six NAC genes (PNAC007, 018, 019, 052, 082 and 163) did not have their corresponding probe sets in the dataset, and the expression profiles of the rest of 157 NAC genes were analyzed as indicated in Figure 7. The majority of NAC genes showed distinct tissue-specific expression patterns across the nine tissues examined (Figure 7 and 
Additional file 9). It is notably that a relatively large fraction of NAC genes, accounting for more than $40 \%$ (67 of 163), were preferentially expressed in male and female catkins. Among these genes, thirty-four gene (50\%) showed the highest transcript abundances in male catkins, twelve (18\%) had the highest accumulation in female catkins, and the remaining 21 (32\%) showed approximately equal transcript abundances in both male and female catkins. The number of NAC genes fell into this category is significantly higher compared to that of MYB transcription factor gene family analyzed by the same microarray data, in which, fifty (28\%) R2R3-MYB genes showed the highest transcript accumulation in catkins [61]. The large proportion of NAC genes with the highest transcript abundance in catkins may probably attribute to the unique characteristic of sex determination in Populus lineage. The previous phylogenetic analysis revealed that these catkin-specific genes were dispersed among different subfamilies rather than divided into distinct subgroups (Figure 1).

Thirteen (8\%) NAC genes showed the highest transcript abundances in differentiating xylems, including four xylem-specific NACs (PNAC070, 086, 121 and 122) identified in the previous microarray analysis (Figure 6C). The six well-characterized NAC genes involved in the secondary cell wall thickening in the previous studies were all included in this category [19]. The expression pattern of PNAC008 gene was inconsistent with the above microarray analysis (Figure 6). The current data revealed the highest expression of PNAC008 gene in young leaves and female catkins rather than in differentiating xylems. The number of NAC genes with the highest transcript abundances in differentiating xylems seems to be a little bit lower than that of R2R3 MYB gene family, which has 23 members accounting for about 12 percent of the total [61]. In addition, a considerable fraction of 24 (15\%) NAC genes were specifically expressed in roots.

Interestingly, a subset of NAC genes demonstrated different expression patterns between dark-grown etiolated seedlings and continuous light grown seedlings, suggesting that these NACs may be subject to photoperiodic regulation. Strikingly, the majority of NAC genes did not show seemingly high abundances of transcript in either young or mature leaves as depicted by the previous ESTs digital profiles analysis (Figure 4 and Additional file 7). One possible explanation may lie in the fact of different sample size and coverage exemplified in the analyses.

The high proportion of segmental duplication of NAC genes and the preferential retention of duplicates raises the question about their functional redundancy. Duplicate genes may have different evolutionary fates: nonfunctionalization, neofunctionalization, or subfunctionalization [76], which may be indicated with divergence in expression patterns. Of the 49 homologous pairs of NAC genes, six genes (PNAC007, 018, 019, 052, 082 and 163) did not have corresponding probe sets on Affymetrix microarray and were excluded for further analysis. Therefore, the remaining 44 homologous pairs were analyzed. Among them, nineteen pairs were located onto duplicated blocks and three pairs were tandem duplicates. Five pairs (PNAC004/006, PNAC012/013, PNAC084/085, PNAC129/130 and PNAC144/145) out of 19 segmental duplications shared the same expression patters with respect to the tissues examined (Figure 7). In addition, the expression pattern of homologous pair PNAC004/006 was also supported by a broad sample sets from the previously microarray and ESTs profiles (Figure 4). Although the expression patterns of the rest of 14 duplicate genes were partially redundant, distinct pattern shifts can be discerned, which suggested that they have undergone subfunctionalization. These findings indicated that expression patterns of NACs have diverged quickly after segmental duplications, thus the NAC genes in Populus are likely to have been retained by substantial subfunctionalization. For example, PNAC008 gene were mainly expressed in young leaves and male catkins, whereas its duplicate counterpart PNAC010 gene extended to a broader expression patterns in young and mature leaves, female and male catkins (Figure 7).

Surprisingly, two homologous pairs (PNAC049/050 and PNAC121/122) also exhibited almost identical patterns of transcript accumulation but were located onto different duplication blocks (Figure 7). The reasons underlying their highly identical expression profiles remain to be investigated since we could not draw any clues from the segmental duplication events. The rest ten homologous pairs also showed almost identical expression patterns, but we could not locate them onto duplicated blocks currently since some of them were mapped to not-yet assembled scaffolds.

As for the tandem duplicated clusters with three genes, the expression patterns of two of them (PNAC020/022, PNAC033/035, PNAC141/143) were almost identical, while significant diversification was observed for the third member, which may indicate neofunctionalization. For example, tandem duplicate PNAC020 and PNAC022 were predominantly expressed in male catkins followed by roots, while PNAC030 was largely expressed in root followed by female catkin (Figure 7). As for the tandem duplicated clusters with two genes, their expression patterns diversified significantly, indicative of substantial neofunctionalization during subsequent evolution processes.

\section{Validation of NAC gene expressions by quantitative real- time RT-PCR}

To verify the expression profiles of Populus NAC genes obtained by in silico EST and microarray data analysis, 


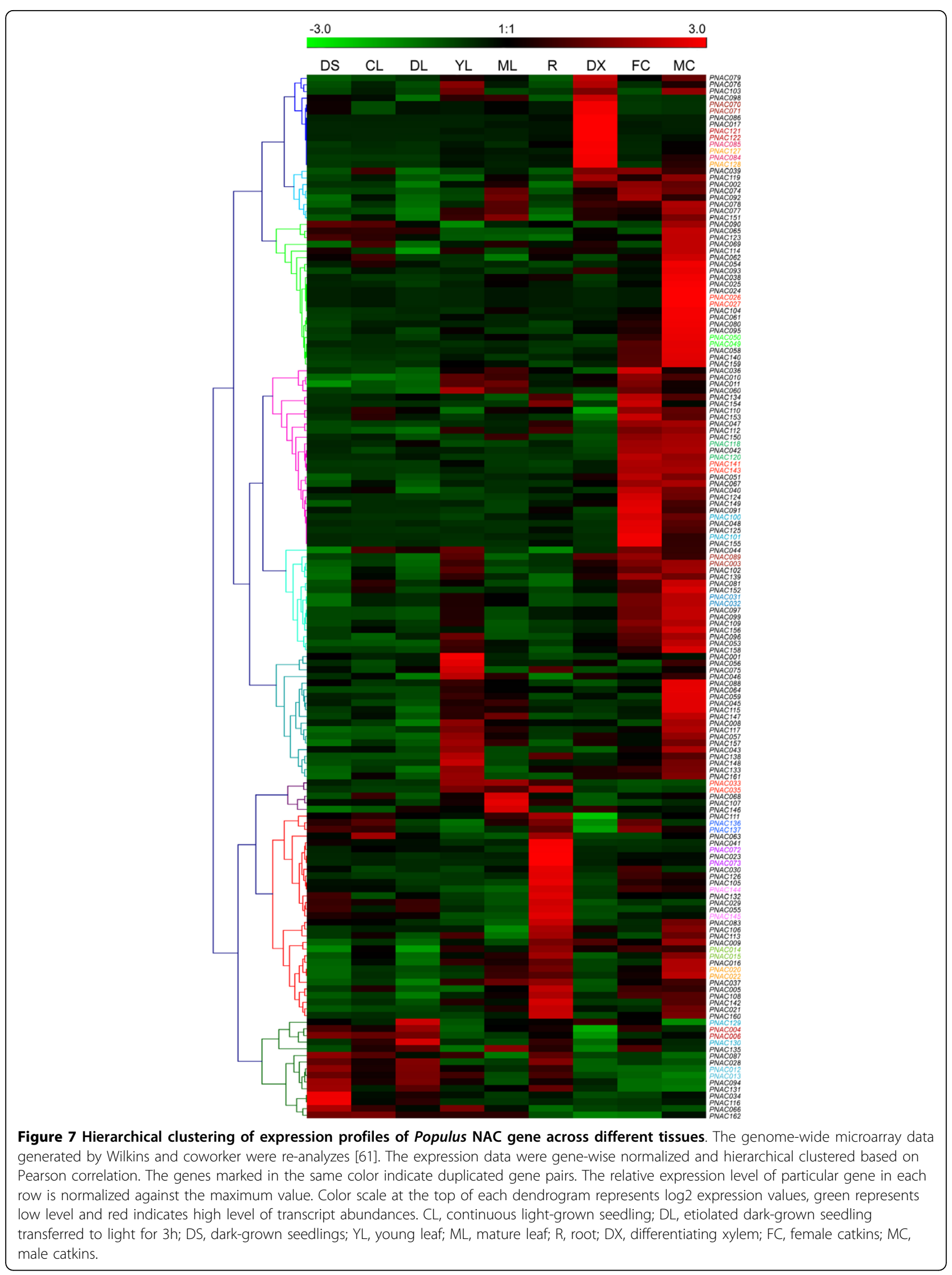




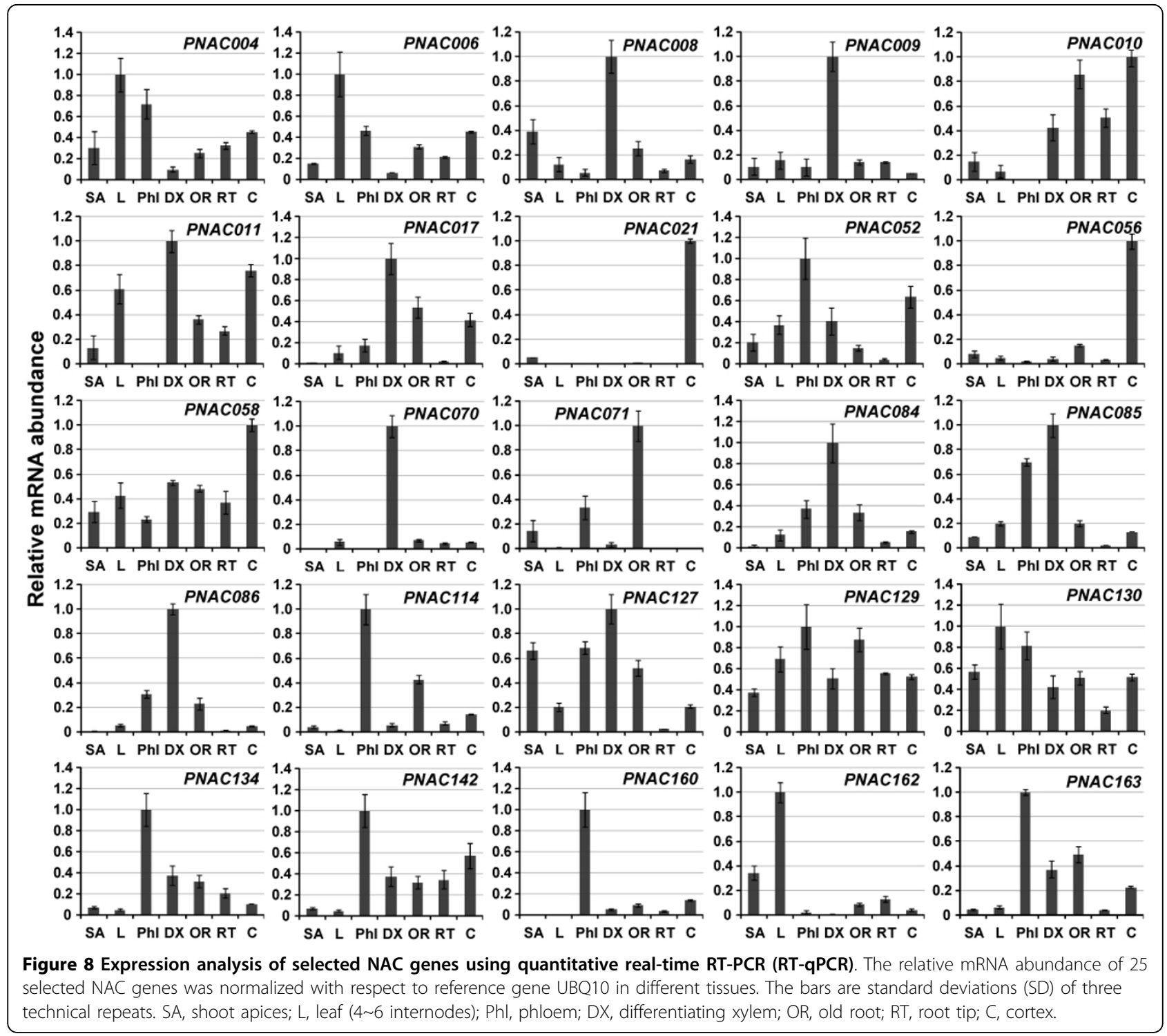

quantitative real-time RT-PCR (RT-qPCR) was performed on seven different tissues for 25 selected NAC genes. As illustrated in Figure 8, the genes showed very distinct tissue-specific expression patterns, which were in good agreement with the above microarray and EST profiles (Figure 6, 7). For example, in accordance with previous findings, a subset of eight genes among the 25 NACs tested were exclusively expressed in differentiating xylem, indicative of their putative roles in secondary cell wall formation. Three genes namely PNAC009 (PtWND4B), PNAC071 (PtWND1B) and PNAC085 (PtWND2B) have been functionally characterized in a recent report [19]. In the present study, PNAC071 gene was shown to primarily express in old root, while microarray profiles indicated the highest transcript abundance in differentiating xylems (Figure 7). Seven genes showed the highest transcript levels in phloem and four genes had the most abundant transcripts in cortex, which were both enriched in primary cell walls. These genes may have putative roles in primary cell wall biosynthesis and wood development. Consistent with the microarray profiles, four genes (PNAC004, 006, 130 and 162) were expressed specifically in leaves. Accordingly, PNAC004 gene had the largest number (up to 24) of corresponding EST sequences originated from leaves, which lent further supports to the above statement. All the 25 genes tested have constitutively weak expression levels in root meristem. Since only a few numbers of NAC genes have been functionally characterized up to date, our results presented here may provide some clues for the selection of candidate genes for further characterization. 


\section{Conclusion}

In the present study, a comprehensive analysis including phylogeny, chromosomal location, gene structure, conserved motifs, and expression profiling of NAC gene family in Populus was performed. We identified a sum of 163 full-length NAC genes in Populus genome, and they were phylogeneticly clustered into 18 distinct subfamilies. The exon/intron structure and motif compositions of NACs were highly conserved in each subfamily, indicative of their functional conservation. The NAC genes were non-randomly distributed across 19 LGs, and a high proportion of NACs were preferentially retained duplicates located on the duplicated blocks, which indicated that segmental duplications contribute significantly to the expansion of Populus NAC gene family. Although a majority of NAC genes showed specific temporal and spatial expression patterns based on EST frequency and microarray data analyses, the expression patterns of a considerable proportion of duplicate genes (14 of 44) were partially redundant, suggesting the occurrence of subfunctionalization during evolutionary process. In other words, a fraction of NAC genes in Populus are likewise to have been retained by substantial subfunctionalization. Furthermore, we identified a subset of Populus NAC genes with putative functional roles in wood-forming and secondary cell wall biosynthesis, and results presented here will be helpful for future functional studies to unravel their divergent roles.

\section{Methods}

\section{Database search and sequence retrieval}

The $P$. trichocarpa genome sequences were downloaded from Phytozome http://www.phytozome.net/poplar. Hidden Markov Model (HMM) profile of NAC domain (PF02365) downloaded from Protein family (Pfam) http://pfam.sanger.ac.uk/ was exploited for the identification of the NAC genes from Populus genome using HMMER (v 2.3.2) [89]. All non-redundant hits with expected values less than 1.0 were collected and then compared with the NAC family in PlnTFDB http:// plntfdb.bio.uni-potsdam.de/v3.0/[3] and DPTF http:// dptf.cbi.pku.edu.cn/[58]. As for the incorrectly predicted genes, manual reannotation was performed using online web server FGENESH http://linux1.softberry.com/berry. phtml[59]. The reannotated sequences were further manually analyzed to confirm the presence of NAM domain using InterProScan program http://www.ebi.ac. uk/Tools/InterProScan/[60]. Sequences of Arabidopsis and rice NAC domain proteins were downloaded from the Arabidopsis genome TAIR 9.0 release http://www. Arabidopsis.org/ and rice genome annotation database (http://rice.plantbiology.msu.edu/, release 5.0), respectively.

\section{Phylogenetic analysis}

Multiple sequence alignments of the full-length protein sequences, including the highly conserved $\mathrm{N}$-terminal NAM domain and the more divergent C-terminal activation domain, were performed by Clustal $\mathrm{X}$ (version 1.83) program [90]. The unrooted phylogenetic trees were constructed with MEGA 4.0 using the NeighborJoining (NJ), Minimal Evolution (ME) and Maximum Parsimony (MP) methods and the bootstrap test carried out with 1000 iterations [91]. Pairwise gap deletion mode was used to ensure that the more divergent $\mathrm{C}$-terminal domains could contribute to the topology of the NJ tree.

\section{Genomic structure and chromosomal location}

Gene structure display server (GSDS) program [92] was used to illustrate exon/intron organization for individual NAC genes by comparison of the cDNAs with their corresponding genomic DNA sequences from Phytozome http://www.phytozome.net/poplar. Identification of homeologous chromosome segments resulting from whole-genome duplication events was accomplished as described in Tuskan et al. (2006) [5]. Blocks of the same color represent the homologous chromosome segments. The tandem gene duplications in Populus were identified according to the same criteria described in rice [93]. Genes separated by five or fewer gene loci in a range of $100 \mathrm{~kb}$ distance were considered to be tandem duplicates.

\section{Identification of conserved motifs}

The program MEME version 4.3.0 was used for the elucidation of motifs in 163 deduced Populus NAC protein sequences http://meme.sdsc.edu[94]. MEME was run locally with the following parameters: number of repetitions - any, maximum number of motifs - 20 , and the optimum motif widths were constrained to between 6 and 200 residues. Structural motif annotation was performed using the SMART http://smart.embl-heidelberg. de[95] and Pfam http://pfam.sanger.ac.uk databases [96].

\section{EST profiling and microarray analysis}

A total of 429,444 Populus EST sequences were downloaded from the GenBank database (http://www.ncbi. nlm.nih.gov/est/, release 121009). The coding regions of NAC genes were used as queries to perform a local BLASTN search against all of the ESTs. Matches above $96 \%$ identity and over an alignment of at least $100 \mathrm{bp}$ were considered as corresponding sequences of the NAC genes. The entries identified were manually inspected for their tissue origin. In addition, the DigitalNorthen tool at the PopGenIE http://www.popgenie.org/ was utilized to produce the heat map with dendrograms of the NAC genes with their corresponding gene model IDs. 
Populus microarray data were obtained from PopGenIE ftp://aspnas.fysbot.umu.se/, the expression data were gene-wise normalized and hierarchical clustered based on Pearson coefficients with average linkage in the Genesis (version 1.75) program [97].

The genome-wide microarray data performed by Wilkins and coworkers [61] were obtained from the NCBI Gene Expression Omnibus (GEO) [98] with accession number GSE13990. Probe sets corresponding to the putative Populus NACs were identified using an online Probe Match tool available at the NetAffx Analysis Center http://www.affymetrix.com/. For genes with more than one probe sets, the median of expression values were considered. When several genes have the same probe set, then they are considered to have same level of transcript abundance. the expression data were genewise normalized and hierarchical clustered based on Pearson coefficients with average linkage in the Genesis (version 1.75) program [97].

\section{Plant material and growth conditions}

One-year-old Populus deltoides was grown in the greenhouse under long day conditions ( $16 \mathrm{~h}$ light $/ 8 \mathrm{~h}$ dark) at a temperature $25^{\circ} \mathrm{C} \sim 28^{\circ} \mathrm{C}$. Shoot apices (internodes $1 \sim 3$ from top, same as below), leaf (from internodes 4 6), developing xylem (from the basal internodes), phloem (from the basal internodes), cortex (from the basal internodes), old root and root tip (terminal 3 5 $\mathrm{mm}$ ) tissues were separately harvested. All samples were immediately frozen in liquid nitrogen and stored at $-80^{\circ} \mathrm{C}$ until required.

\section{RNA isolation and quantitative real-time RT-PCR (RT-qPCR)}

Total RNA from the majority of the samples was extracted using TRIzol reagent (Invitrogen, $\mathrm{Ca}, \mathrm{USA}$ ) according to manufacturer's instructions. Alternatively, total RNA from cortex, old root and root tip were isolated by CTAB method [99] with minor modifications. RNA integrity was verified by $2 \%$ agar gel electrophoresis and SYBR Greenlstaining. Before cDNA synthesis, RNA was treated with RQ1 RNase-free DNase (Promega, Madison, WI, USA) according to the manufacturer's instructions to ensure no DNA contamination, and then the first-strand cDNA synthesis was carried out with approximately $2 \mu \mathrm{g}$ RNA using the RevertAid First Strand cDNA Synthesis Kit (MBI, Fermentas) and oilgo-dT primers according to the manufacturer's procedure. Primers were designed using Beacon Designer v7.0 (Premier Biosoft International, Palo Alto, California, USA) with melting temperatures $58 \sim 60^{\circ} \mathrm{C}$, primer lengths 20 24bp and amplicon lengths 51 199bp. Experimental details are given in additional file 10.
RT-qPCR was conducted on LightCycler 480 Detection System (Roche, Penzberg, Germany) using SYBR Premix Ex Taq (TaKaRa, Toyoto, Japan). Reactions were prepared in a total volume of $20 \mu \mathrm{l}$ containing: $2 \mu \mathrm{l}$ of template, $10 \mu \mathrm{l}$ of $2 \times \mathrm{SYBR}$ Premix, $0.4 \mu \mathrm{l}$ of each specific primer to a final concentration of $200 \mathrm{nM}$. The reactions were performed as the following conditions: initial denaturation step of $95^{\circ} \mathrm{C}$ for $10 \mathrm{~s}$ followed by two-step thermal cycling profile of denaturation at $95^{\circ} \mathrm{C}$ for $5 \mathrm{~s}$, and combined primer annealing/extension at $60^{\circ} \mathrm{C}$ for 1 min for 40 cycles. No-template controls were included for each primer pair and each PCR reaction was completed in triplicate. To verify the specificity of the amplicon for each primer pair, a melting curve analysis was performed ranging from $60^{\circ} \mathrm{C}$ to $95^{\circ} \mathrm{C}$ with temperature increasing steps of $0.06^{\circ} \mathrm{C} / \mathrm{s}$ ( 5 acquisitions per ${ }^{\circ} \mathrm{C}$ ) at the end of each PCR run. Baseline and threshold cycles $(\mathrm{Ct})$ were automatically determined using the LightCycler $^{\circ} 480$ Software release 1.5.0. Relative gene expression with respect to internal reference gene UBQ10 was determined as described previously [100].

\section{Acknowledgements}

This study was supported by grants from the National High-Tech Research and Development Program of China (to G.Z., 2009AA10Z101), the Program of 100 Distinguished Young Scientists of the Chinese Academy of Sciences (to G.Z., No. 428) and National Natural Science Foundation of China (No. 30901157).

\section{Additional material}

Additional file 1: A complete list of NAC gene sequences identified in the present study. The list comprises 163 NAC gene sequences identified. Amino acid sequences are deduced from their corresponding coding sequences and genomic DNA sequences are obtained from Phytozome http://www.phytozome.net/poplar, release 2.0.

Additional file 2: Phylogenetic tree of $\mathrm{N}$-terminus NAC domain proteins from Populus, Arabidopsis and rice. The unrooted tree was constructed using MEGA 4.0 with the Neighbor-Joining (NJ) method after alignment of the conserved N-terminus domain of 163 Populus, 105 Arabidopsis and 140 rice NAC genes. Only the tree topology is presented.

Additional file 3: Phylogenetic tree of full-length NAC domain proteins from Populus, Arabidopsis and rice. The unrooted tree was constructed using MEGA 4.0 with the Neighbor-Joining (NJ) method after alignment of the full-length amino acid sequences of 163 Populus, 105 Arabidopsis and 140 rice NAC genes. Numbers at nodes indicate the percentage bootstrap scores and only bootstrap values higher than $50 \%$ from 1000 replicates are shown. The scale bar corresponds to 0.1 estimated amino acid substitutions per site.

Additional file 4: Microarray based expression profiles of Arabidopsis NAC genes across a variety of tissue/organs. Expression of NAC genes during developmental stages are presented as heat maps generated using meta-analysis tool at GENEVESTIGATOR http://www. genevestigator.ethz.ch and clustered using hierarchical clustering with average linkage. The transcript levels are depicted by color scale representing log2 values. Dark blue denotes high expression and light blue denotes low expression. 
Additional file 5: Pairwise identities between homologous pairs of NAC genes from Populus. Pairwise identities and sequence alignments of the 49 homologous pairs identified from Populus NACs.

Additional file 6: Exon/intron organization of Populus NAC genes. Exons and introns are represented by green boxes and black lines respectively. The numbers indicate the splicing phases of the NAC genes, 0 refers to phase 0,1 to phase 1 , and 2 to phase 2 .

Additional file 7: Sequence logos for the conserved motifs of Populus NAC domain proteins. Conserved motifs and the sequence logos were generated using the MEME search tool. Numbers on the horizontal axis represent the sequence positions in the motifs and the vertical axis represents the information content measured in bits. Motif 2 represents the NAM sub-domain A, motif 5 represents the NAM subdomain B, motif 1 represents the NAM sub-domain C, motif 3 represents the NAM sub-domain D, and motif 6 represents the NAM sub-domain E.

Additional file 8: Expression proflies of Populus NAC genes revealed by clustering analysis of the digital northern data. The

DigitalNorthen tool at the PopGenlE http://www.popgenie.org/ was utilized to produce the heat map with dendrograms of the NAC genes. Color bar at bottom represents the frequencies of EST counts.

Additional file 9: Raw data from microarray expression analyses for Populus NAC genes. Sample abbreviations are defined in Figure 6 and Figure 7.

Additional file 10: Primer sequences of the selected NAC genes for RT-qPCR analysis. A list of primer sequences of the 25 selected NAC gens for RT-qPCR assay.

\section{Author details}

'Qingdao Institute of BioEnergy and Bioprocess Technology, Chinese Academy of Sciences, Qingdao 266101, PR China. ${ }^{2}$ Current address: Complex Carbohydrate Research Center, The University of Georgia, 315 Riverbend Road, Athens, GA 30602, USA.

\section{Authors' contributions}

$\mathrm{RH}, \mathrm{GQ}$ and $\mathrm{YK}$ performed the computational analysis, expression analyses and drafted the manuscript cooperatively. DK and QG participated in the data mining, helped in Populus materials collection and data analysis. GZ conceived the project, supervised the analysis and critically revised the manuscript. All authors read and approved the final manuscript.

Received: 8 February 2010 Accepted: 15 July 2010

Published: 15 July 2010

\section{References}

1. Riechmann JL, Heard J, Martin G, Reuber L, Jiang C, Keddie J, Adam L, Pineda O, Ratcliffe OJ, Samaha RR, Creelman R, Pilgrim M, Broun P, Zhang JZ, Ghandehari D, Sherman BK, Yu G: Arabidopsis transcription factors: genome-wide comparative analysis among eukaryotes. Science 2000, 290(5499):2105-2110

2. Wray GA, Hahn MW, Abouheif E, Balhoff JP, Pizer M, Rockman MV Romano LA: The evolution of transcriptional regulation in eukaryotes. Mol Biol Evol 2003, 20(9):1377-1419.

3. Riano-Pachon DM, Ruzicic S, Dreyer I, Mueller-Roeber B: PInTFDB: an integrative plant transcription factor database. BMC Bioinformatics 2007, $8: 42$.

4. AtTFDB - Arabidopsis transcription factor database. [http://arabidopsis. med.ohio-state.edu/AtTFDB/.

5. Tuskan GA, Difazio S, Jansson S, Bohlmann J, Grigoriev I, Hellsten U, Putnam N, Ralph S, Rombauts S, Salamov A, Schein J, Sterck L, Aerts A, Bhalerao RR, Bhalerao RP, Blaudez D, Boerjan W, Brun A, Brunner A, Busov V, Campbell M, Carlson J, Chalot M, Chapman J, Chen GL, Cooper D, Coutinho PM, Couturier J, Covert S, Cronk Q, et al: The genome of black cottonwood, Populus trichocarpa (Torr. \& Gray). Science 2006, 313(5793):1596-1604.

6. Ooka H, Satoh K, Doi K, Nagata T, Otomo Y, Murakami K, Matsubara K, Osato N, Kawai J, Carninci P, Hayashizaki Y, Suzuki K, Kojima K, Takahara Y,
Yamamoto K, Kikuchi S: Comprehensive analysis of NAC family genes in Oryza sativa and Arabidopsis thaliana. DNA Res 2003, 10(6):239-247.

7. Fang Y, You J, Xie K, Xie W, Xiong L: Systematic sequence analysis and identification of tissue-specific or stress-responsive genes of NAC transcription factor family in rice. Mol Genet Genomics 2008, 280(6):547-563.

8. Pinheiro GL, Marques CS, Costa MD, Reis PA, Alves MS, Carvalho CM, Fietto $L G$, Fontes EP: Complete inventory of soybean NAC transcription factors: sequence conservation and expression analysis uncover their distinct roles in stress response. Gene 2009, 444(1-2):10-23.

9. Kikuchi K, Ueguchi-Tanaka M, Yoshida KT, Nagato Y, Matsusoka M, Hirano HY: Molecular analysis of the NAC gene family in rice. Mol Gen Genet 2000, 262(6):1047-1051.

10. Aida M, Ishida T, Fukaki H, Fujisawa H, Tasaka M: Genes involved in organ separation in Arabidopsis: an analysis of the cup-shaped cotyledon mutant. Plant Cell 1997, 9(6):841-857.

11. Duval M, Hsieh TF, Kim SY, Thomas TL: Molecular characterization of AtNAM: a member of the Arabidopsis NAC domain superfamily. Plant Mol Biol 2002, 50(2):237-248.

12. Ernst HA, Olsen AN, Larsen S, Lo Leggio L: Structure of the conserved domain of ANAC, a member of the NAC family of transcription factors. EMBO Rep 2004, 5(3):297-303.

13. Hegedus D, Yu M, Baldwin D, Gruber M, Sharpe A, Parkin I, Whitwill S, Lydiate D: Molecular characterization of Brassica napus NAC domain transcriptional activators induced in response to biotic and abiotic stress. Plant Mol Biol 2003, 53(3):383-397.

14. Olsen AN, Ernst HA, Leggio LL, Skriver K: NAC transcription factors: structurally distinct, functionally diverse. Trends Plant Sci 2005, 10(2):79-87.

15. Xie Q, Frugis $\mathrm{G}$, Colgan $\mathrm{D}$, Chua NH: Arabidopsis NAC1 transduces auxin signal downstream of TIR1 to promote lateral root development. Genes Dev 2000, 14(23):3024-3036.

16. Jensen MK, Kjaersgaard T, Nielsen MM, Galberg P, Petersen K, O'Shea C, Skriver $K$ : The Arabidopsis thaliana NAC transcription factor family: structure-function relationships and determinants of ANAC019 stress signaling. Biochem J 2009.

17. Ko JH, Yang SH, Park AH, Lerouxel O, Han KH: ANAC012, a member of the plant-specific NAC transcription factor family, negatively regulates xylary fiber development in Arabidopsis thaliana. Plant J 2007, 50(6):1035-1048.

18. Fujita M, Fujita Y, Maruyama K, Seki M, Hiratsu K, Ohme-Takagi M, Tran LS, Yamaguchi-Shinozaki K, Shinozaki K: A dehydration-induced NAC protein, $\mathrm{RD} 26$, is involved in a novel ABA-dependent stress-signaling pathway. Plant J 2004, 39(6):863-876.

19. Zhong R, Lee C, Ye ZH: Functional Characterization of Poplar WoodAssociated NAC Domain Transcription Factors. Plant Physiol 2009.

20. Zheng $X$, Chen B, Lu G, Han B: Overexpression of a NAC transcription factor enhances rice drought and salt tolerance. Biochem Biophys Res Commun 2009, 379(4):985-989.

21. Yamaguchi M, Kubo M, Fukuda H, Demura T: Vascular-related NACDOMAIN7 is involved in the differentiation of all types of xylem vessels in Arabidopsis roots and shoots. Plant J 2008, 55(4):652-664.

22. Taoka K, Yanagimoto Y, Daimon Y, Hibara K, Aida M, Tasaka M: The NAC domain mediates functional specificity of CUP-SHAPED COTYLEDON proteins. Plant J 2004, 40(4):462-473.

23. Souer $E$, van Houwelingen A, Kloos D, Mol J, Koes R: The no apical meristem gene of Petunia is required for pattern formation in embryos and flowers and is expressed at meristem and primordia boundaries. Cell 1996, 85(2):159-170.

24. Weir I, Lu J, Cook H, Causier B, Schwarz-Sommer Z, Davies B: CUPULIFORMIS establishes lateral organ boundaries in Antirrhinum. Development 2004, 131(4):915-922.

25. Hibara K, Takada S, Tasaka M: CUC1 gene activates the expression of SAM-related genes to induce adventitious shoot formation. Plant $J 2003$, 36(5):687-696

26. Nikovics K, Blein T, Peaucelle A, Ishida T, Morin H, Aida M, Laufs P: The balance between the MIR164A and CUC2 genes controls leaf margin serration in Arabidopsis. Plant Cell 2006, 18(11):2929-2945.

27. Sablowski RW, Meyerowitz EM: A homolog of NO APICAL MERISTEM is an immediate target of the floral homeotic genes APETALA3/PISTILLATA. Cell 1998, 92(1):93-103.

28. He XJ, Mu RL, Cao WH, Zhang ZG, Zhang JS, Chen SY: AtNAC2, a transcription factor downstream of ethylene and auxin signaling 
pathways, is involved in salt stress response and lateral root development. Plant J 2005, 44(6):903-916.

29. Guo Y, Gan S: AtNAP, a NAC family transcription factor, has an important role in leaf senescence. Plant J 2006, 46(4):601-612.

30. Uauy C, Distelfeld A, Fahima T, Blechl A, Dubcovsky J: A NAC Gene regulating senescence improves grain protein, zinc, and iron content in wheat. Science 2006, 314(5803):1298-1301.

31. Yoo SY, Kim Y, Kim SY, Lee JS, Ahn JH: Control of flowering time and cold response by a NAC-domain protein in Arabidopsis. PLoS One 2007, 2(7): e642.

32. Kim SG, Kim SY, Park CM: A membrane-associated NAC transcription factor regulates salt-responsive flowering via FLOWERING LOCUS T in Arabidopsis. Planta 2007, 226(3):647-654.

33. Kim YS, Kim SG, Park JE, Park HY, Lim MH, Chua NH, Park CM: A membrane-bound NAC transcription factor regulates cell division in Arabidopsis. Plant Cell 2006, 18(11):3132-3144

34. Willemsen V, Bauch M, Bennett T, Campilho A, Wolkenfelt $H, X u$ J, Haseloff J, Scheres B: The NAC domain transcription factors FEZ and SOMBRERO control the orientation of cell division plane in Arabidopsis root stem cells. Dev Cell 2008, 15(6):913-922.

35. Kato H, Motomura T, Komeda Y, Saito T, Kato A: Overexpression of the NAC transcription factor family gene ANAC036 results in a dwarf phenotype in Arabidopsis thaliana. J Plant Physiol 2009

36. Jensen MK, Hagedorn PH, de Torres-Zabala M, Grant MR, Rung JH, Collinge DB, Lyngkjaer MF: Transcriptional regulation by an NAC (NAMATAF1,2-CUC2) transcription factor attenuates ABA signalling for efficient basal defence towards Blumeria graminis $\mathrm{f}$. $\mathrm{sp}$. hordei in Arabidopsis. Plant J 2008, 56(6):867-880.

37. Waters BM, Uauy C, Dubcovsky J, Grusak MA: Wheat (Triticum aestivum) NAM proteins regulate the translocation of iron, zinc, and nitrogen compounds from vegetative tissues to grain. J Exp Bot 2009, 60(15):4263-4274.

38. Mao C, Ding W, Wu Y, Yu J, He X, Shou H, Wu P: Overexpression of a NAC-domain protein promotes shoot branching in rice. New Phytol 2007, 176(2):288-298.

39. Hu H, Dai M, Yao J, Xiao B, Li X, Zhang Q, Xiong L: Overexpressing a NAM ATAF, and CUC (NAC) transcription factor enhances drought resistance and salt tolerance in rice. Proc Natl Acad Sci USA 2006, 103(35):12987-12992

40. Tran LS, Nakashima K, Sakuma Y, Simpson SD, Fujita Y, Maruyama K, Fujita M, Seki M, Shinozaki K, Yamaguchi-Shinozaki K: Isolation and functional analysis of Arabidopsis stress-inducible NAC transcription factors that bind to a drought-responsive cis-element in the early responsive to dehydration stress 1 promoter. Plant Cell 2004, 16(9):2481-2498

41. Jiang $Y$, Deyholos MK: Comprehensive transcriptional profiling of $\mathrm{NaCl}-$ stressed Arabidopsis roots reveals novel classes of responsive genes. BMC Plant Biol 2006, 6:25

42. Collinge $M$, Boller T: Differential induction of two potato genes, Stprx2 and StNAC, in response to infection by Phytophthora infestans and to wounding. Plant Mol Biol 2001, 46(5):521-529.

43. Ren T, Qu F, Morris TJ: HRT gene function requires interaction between a NAC protein and viral capsid protein to confer resistance to turnip crinkle virus. Plant Cell 2000, 12(10):1917-1926.

44. Delessert C, Kazan K, Wilson IW, Van Der Straeten D, Manners J, Dennis ES, Dolferus R: The transcription factor ATAF2 represses the expression of pathogenesis-related genes in Arabidopsis. Plant J 2005, 43(5):745-757.

45. Kim SG, Lee AK, Yoon HK, Park CM: A membrane-bound NAC transcription factor NTL8 regulates gibberellic acid-mediated salt signaling in Arabidopsis seed germination. Plant J 2008, 55(1):77-88

46. Lu PL, Chen NZ, An R, Su Z, Qi BS, Ren F, Chen J, Wang XC: A novel drought-inducible gene, ATAF1, encodes a NAC family protein that negatively regulates the expression of stress-responsive genes in Arabidopsis. Plant Mol Biol 2007, 63(2):289-305.

47. Wang X, Basnayake BM, Zhang H, Li G, Li W, Virk N, Mengiste T, Song F: The Arabidopsis ATAF1, a NAC transcription factor, is a negative regulator of defense responses against necrotrophic fungal and bacterial pathogens. Mol Plant Microbe Interact 2009, 22(10):1227-1238.

48. Zhao C, Avci U, Grant EH, Haigler CH, Beers EP: XND1, a member of the $\mathrm{NAC}$ domain family in Arabidopsis thaliana, negatively regulates lignocellulose synthesis and programmed cell death in xylem. Plant $J$ 2008, 53(3):425-436.

49. Zhao C, Craig JC, Petzold HE, Dickerman AW, Beers EP: The xylem and phloem transcriptomes from secondary tissues of the Arabidopsis roothypocotyl. Plant Physiol 2005, 138(2):803-818.

50. Zhong R, Demura T, Ye ZH: SND1, a NAC domain transcription factor, is a key regulator of secondary wall synthesis in fibers of Arabidopsis. Plant Cell 2006, 18(11):3158-3170

51. Zhong R, Lee C, Zhou J, McCarthy RL, Ye ZH: A battery of transcription factors involved in the regulation of secondary cell wall biosynthesis in Arabidopsis. Plant Cell 2008, 20(10):2763-2782.

52. Zhong $\mathrm{R}$, Richardson EA, Ye ZH: Two NAC domain transcription factors, SND1 and NST1, function redundantly in regulation of secondary wall synthesis in fibers of Arabidopsis. Planta 2007, 225(6):1603-1611.

53. Mitsuda N, Iwase A, Yamamoto H, Yoshida M, Seki M, Shinozaki K, OhmeTakagi M: NAC transcription factors, NST1 and NST3, are key regulators of the formation of secondary walls in woody tissues of Arabidopsis. Plant Cell 2007, 19(1):270-280.

54. Mitsuda N, Ohme-Takagi M: NAC transcription factors NST1 and NST3 regulate pod shattering in a partially redundant manner by promoting secondary wall formation after the establishment of tissue identity. Plant J 2008, 56(5):768-778.

55. Mitsuda N, Seki M, Shinozaki K, Ohme-Takagi M: The NAC transcription factors NST1 and NST2 of Arabidopsis regulate secondary wall thickenings and are required for anther dehiscence. Plant Cell 2005, 17(11):2993-3006.

56. Kubo M, Udagawa M, Nishikubo N, Horiguchi G, Yamaguchi M, Ito J, Mimura T, Fukuda H, Demura T: Transcription switches for protoxylem and metaxylem vessel formation. Genes Dev 2005, 19(16):1855-1860.

57. Shen $H$, Yin $Y B, C$ Chen $F, X u Y$, Dixon RA: A Bioinformatic Analysis of NAC Genes for Plant Cell Wall Development in Relation to Lignocellulosic Bioenergy Production. Bioenerg Res 2009, 2:217-232.

58. Zhu QH, Guo AY, Gao G, Zhong YF, Xu M, Huang M, Luo J: DPTF: a database of poplar transcription factors. Bioinformatics 2007 23(10):1307-1308.

59. Salamov AA, Solovyev $\mathrm{W}$ : Ab initio gene finding in Drosophila genomic DNA. Genome Res 2000, 10(4):516-522

60. Quevillon E, Silventoinen V, Pillai S, Harte N, Mulder N, Apweiler R, Lopez R: InterProScan: protein domains identifier. Nucleic Acids Res 2005, , 33 Web Server: W116-120.

61. Wilkins O, Nahal H, Foong J, Provart NJ, Campbell MM: Expansion and diversification of the Populus R2R3-MYB family of transcription factors. Plant Physiol 2009, 149(2):981-993.

62. Li X, Duan X, Jiang H, Sun Y, Tang Y, Yuan Z, Guo J, Liang W, Chen L, Yin J, Ma H, Wang J, Zhang D: Genome-wide analysis of basic/helix-loop-helix transcription factor family in rice and Arabidopsis. Plant Physiol 2006, 141(4):1167-1184.

63. Lijavetzky D, Carbonero P, Vicente-Carbajosa J: Genome-wide comparative phylogenetic analysis of the rice and Arabidopsis Dof gene families. BMC Evol Biol 2003, 3:17.

64. Wang D, Guo Y, Wu C, Yang G, Li Y, Zheng C: Genome-wide analysis of $\mathrm{CCCH}$ zinc finger family in Arabidopsis and rice. BMC Genomics 2008, 9:44.

65. Zhang $Y$, Wang $L$ : The WRKY transcription factor superfamily: its origin in eukaryotes and expansion in plants. BMC Evol Biol 2005, 5(1):1

66. Nakashima K, Tran LS, Van Nguyen D, Fujita M, Maruyama K, Todaka D, Ito Y, Hayashi N, Shinozaki K, Yamaguchi-Shinozaki K: Functional analysis of a NAC-type transcription factor OsNAC6 involved in abiotic and biotic stress-responsive gene expression in rice. Plant J 2007, 51(4):617-630.

67. Zimmermann P, Hirsch-Hoffmann M, Hennig L, Gruissem W: GENEVESTIGATOR. Arabidopsis microarray database and analysis toolbox. Plant Physiol 2004, 136(1):2621-2632.

68. Brunner AM, Busov VB, Strauss SH: Poplar genome sequence: functional genomics in an ecologically dominant plant species. Trends Plant Sci 2004, 9(1):49-56.

69. Cannon SB, Mitra A, Baumgarten A, Young ND, May G: The roles of segmental and tandem gene duplication in the evolution of large gene families in Arabidopsis thaliana. BMC Plant Biol 2004, 4:10.

70. Barakat A, Bagniewska-Zadworna A, Choi A, Plakkat U, DiLoreto DS, Yellanki P, Carlson JE: The cinnamyl alcohol dehydrogenase gene family 
in Populus: phylogeny, organization, and expression. BMC Plant Biol 2009, 9:26.

71. Kalluri UC, Difazio SP, Brunner AM, Tuskan GA: Genome-wide analysis of Aux/IAA and ARF gene families in Populus trichocarpa. BMC Plant Biol 2007, 7:59.

72. Lan T, Yang ZL, Yang X, Liu YJ, Wang XR, Zeng QY: Extensive Functional Diversification of the Populus Glutathione S-Transferase Supergene Family. Plant Cell 2009.

73. Blanc G, Wolfe KH: Functional divergence of duplicated genes formed by polyploidy during Arabidopsis evolution. Plant Cell 2004, 16(7):1679-1691.

74. Blanc G, Wolfe KH: Widespread paleopolyploidy in model plant species inferred from age distributions of duplicate genes. Plant Cell 2004, 16(7):1667-1678

75. Maere S, De Bodt S, Raes J, Casneuf T, Van Montagu M, Kuiper M, Van de Peer $Y$ : Modeling gene and genome duplications in eukaryotes. Proc Natl Acad Sci USA 2005, 102(15):5454-5459.

76. Prince VE, Pickett FB: Splitting pairs: the diverging fates of duplicated genes. Nat Rev Genet 2002, 3(11):827-837.

77. Vandepoele K, Simillion C, Van de Peer Y: Evidence that rice and other cereals are ancient aneuploids. Plant Cell 2003, 15(9):2192-2202.

78. Kolkman JA, Stemmer WP: Directed evolution of proteins by exon shuffling. Nat Biotechnol 2001, 19(5):423-428.

79. Pfam. [http://pfam.sanger.ac.uk/search].

80. SMART. [http://smart.embl-heidelberg.de/].

81. Ohlrogge J, Benning C: Unraveling plant metabolism by EST analysis. Curr Opin Plant Biol 2000, 3(3):224-228.

82. PopGenIE. [http://www.popgenie.org/].

83. Sjodin A, Bylesjo M, Skogstrom O, Eriksson D, Nilsson P, Ryden P, Jansson S, Karlsson J: UPSC-BASE-Populus transcriptomics online. Plant J 2006, 48(5):806-817.

84. Sjodin A, Street NR, Sandberg G, Gustafsson P, Jansson S: The Populus Genome Integrative Explorer (PopGenIE): a new resource for exploring the Populus genome. New Phytol 2009

85. Druart N, Johansson A, Baba K, Schrader J, Sjodin A, Bhalerao RR, Resman L, Trygg J, Moritz T, Bhalerao RP: Environmental and hormonal regulation of the activity-dormancy cycle in the cambial meristem involves stagespecific modulation of transcriptional and metabolic networks. Plant J 2007, 50(4):557-573.

86. Schrader J, Moyle R, Bhalerao R, Hertzberg M, Lundeberg J, Nilsson P, Bhalerao RP: Cambial meristem dormancy in trees involves extensive remodelling of the transcriptome. Plant J 2004, 40(2):173-187.

87. Moreau C, Aksenov N, Lorenzo MG, Segerman B, Funk C, Nilsson P, Jansson S, Tuominen H: A genomic approach to investigate developmental cell death in woody tissues of Populus trees. Genome Biol 2005, 6(4):R34.

88. Schrader J, Nilsson J, Mellerowicz E, Berglund A, Nilsson P, Hertzberg M, Sandberg G: A high-resolution transcript profile across the wood-forming meristem of poplar identifies potential regulators of cambial stem cell identity. Plant Cell 2004, 16(9):2278-2292.

89. HMMER. [http://hmmer.janelia.org/]

90. Thompson JD, Gibson TJ, Plewniak F, Jeanmougin F, Higgins DG: The CLUSTAL_X windows interface: flexible strategies for multiple sequence alignment aided by quality analysis tools. Nucleic Acids Res 1997 , 25(24):4876-4882.

91. Tamura K, Dudley J, Nei M, Kumar S: MEGA4: Molecular Evolutionary Genetics Analysis (MEGA) software version 4.0. Mol Biol Evol 2007, 24(8):1596-1599.

92. Guo AY, Zhu QH, Chen $X$, Luo JC: [GSDS: a gene structure display server]. Yi Chuan 2007, 29(8):1023-1026.

93. TIGR Rice Genome Annotation. [http://compbio.dfci.harvard.edu/tgi/].

94. Bailey TL, Williams N, Misleh C, Li WW: MEME: discovering and analyzing DNA and protein sequence motifs. Nucleic Acids Res 2006, , 34 Web Server: W369-373.

95. Letunic I, Copley RR, Schmidt S, Ciccarelli FD, Doerks T, Schultz J, Ponting CP, Bork P: SMART 4.0: towards genomic data integration. Nucleic Acids Res 2004, 32 Database: D142-144.

96. Finn RD, Mistry J, Schuster-Bockler B, Griffiths-Jones S, Hollich V, Lassmann T, Moxon S, Marshall M, Khanna A, Durbin R, Eddy SR, Sonnhammer EL, Bateman A: Pfam: clans, web tools and services. Nucleic Acids Res 2006, , 34 Database: D247-251.
97. Sturn A, Quackenbush J, Trajanoski Z: Genesis: cluster analysis of microarray data. Bioinformatics 2002, 18(1):207-208.

98. Gene Expression Omnibus. [http://www.ncbi.nlm.nih.gov/geo/]

99. Chang S, Puryear J, Cairney J: A simple and efficient method for isolating RNA from pine trees. Plant Molecular Biology Reporter 1993, 11:113-116.

100. Livak KJ, Schmittgen TD: Analysis of relative gene expression data using real-time quantitative PCR and the 2(-Delta Delta C(T)) Method. Methods 2001, 25(4):402-408.

doi:10.1186/1471-2229-10-145

Cite this article as: Hu et al:: Comprehensive Analysis of NAC Domain Transcription Factor Gene Family in Populus trichocarpa. BMC Plant Biology 2010 10:145.

\section{Submit your next manuscript to BioMed Central and take full advantage of:}

- Convenient online submission

- Thorough peer review

- No space constraints or color figure charges

- Immediate publication on acceptance

- Inclusion in PubMed, CAS, Scopus and Google Scholar

- Research which is freely available for redistribution

Submit your manuscript at www.biomedcentral.com/submit
C Biomed Central 